\title{
A Simple, Rapid and Mild One Pot Synthesis of Benzene Ring Acylated and Demethylated Analogues of Harmine under Solvent-free Conditions
}

\author{
Sabira Begum*, Syed Nawazish Ali, Farhat, Syed Imran Hassan and Bina S. Siddiqui
}

H. E. J. Research Institute of Chemistry, International Center for Chemical and Biological Sciences, University of Karachi, Karachi-75270, Pakistan; E-mails: nawazishalii@yahoo.com (Syed Nawazish Ali); farhatzubair@yahoo.com (Farhat); imranhassan2@yahoo.com (Syed Imran Hassan); bina@comsats.net.pk (Bina S. Siddiqui)

\footnotetext{
* Author to whom correspondence should be addressed; E-mail: sabira@khi.comsats.net.pk or dr.sabirabegum@yahoo.com; Tel.: +92-21-4824901; Fax: +92-21-4819018/4819019.
}

Received: 6 May 2008; in revised form: 24 June 2008 / Accepted: 23 July 2008 / Published: 6 August 2008

\begin{abstract}
A simple, rapid, solvent-free, room temperature one pot synthesis of benzene ring acylated and demethylated analogues of harmine using acyl halides/acid anhydrides and $\mathrm{AlCl}_{3}$ has been developed. Eight different acyl halides/acid anhydrides were used in the synthesis. The resulting mixture of products was separated by column chromatography to afford 10- and 12-monoacyl analogues, along with 10,12-diacyl-11-hydroxy products. In five cases the corresponding 10-acyl-11-hydroxy analogues were also obtained. Yields from the eight syntheses (29 products in total) were in the 6-34\% range and all compounds were fully characterized.
\end{abstract}

Keywords: Solvent-free reactions, Friedel-Crafts acylation, $\beta$-carboline, harmine, acylharmine, demethylation. 


\section{Introduction}

Solvent-free chemical syntheses have recently received much attention. These processes are not only environmentally benign, but also economically beneficial because toxic wastes can be minimized or eliminated, so the costs of waste treatment are also reduced. An additional attractive feature is their operational simplicity. Thus, there are now over 500 published papers describing solvent-free reactions giving quantitative yields on the gram and kilogram scale that support the effectiveness of the method [1-4].

Friedel-Crafts acylation is one of the most important protocols for the formation of $\mathrm{C}-\mathrm{C}$ bonds between aromatic rings and aliphatic moieties. This method generally requires addition of a Lewis acid catalyst such as $\mathrm{AlCl}_{3}$ to a well-stirred mixture of an alkyl or acyl halide and an aromatic compound under inert conditions. Usually, these reactions require high temperatures, long reaction times and tedious workups [5-6]. Recently, Friedel-Crafts acylation of alkoxybenzenes was achieved efficiently by a reaction with aliphatic acid anhydrides in the presence of catalytic amounts of aluminum hydrogensulfate, $\mathrm{Al}\left(\mathrm{HSO}_{4}\right)_{3}$, both in nitromethane and under solvent-free conditions, but these reactions required high temperaures $\left(70^{\circ} \mathrm{C}\right)$ for completion [7]. Although the acetylation of aryl ethers using acetic anhydride in the presence of zeolites under mild conditions in a solvent-free system gave the corresponding para-acetylated products in high yields, these reactions also required high temperatures $\left(120^{\circ} \mathrm{C}\right)$ and occurred only in activated aromatic compounds [8]. The use of metal triflates in ionic liquids for Friedel-Crafts acylation have also been reported, but it is again limited to highly activated substrates and also requires high temperatures [9].

The $\beta$-carboline alkaloids, one of which is harmine, are very important natural products due to their interesting chemistry, pharmacological importance and therapeutic potential. They posses antitumor, anti-HIV and other important biological activities [10-12]. Recently, we have reported the Friedel-Crafts acylation of $\mathrm{N}$-acetyltetrahydroharmine under solvent-free conditions, which resulted in the synthesis of a series of its 10-acyl and 12-acyl analogues in high overall yields [13]. Here we would like to present a study of the reaction of the Friedel-Crafts reagents (acyl halides/acid anhydrides and $\mathrm{AlCl}_{3}$ ) with harmine (7-MeO-1-Me-9H-pyrido[3,4-b]-indole, 1), another $\beta$-carboline alkaloid, at room temperature and under solvent-free conditions. The harmine used in these investigations was isolated from Peganum harmala following a method reported earlier [14]. Eight different syntheses were carried out, which yielded 29 different products. None of these products had previously been reported. After separation by column chromatography the reaction mixtures gave 10acyl (2-9), 12-acyl (10-17), 10-acyl-11-hydroxy (18-22) and 10,12-diacyl-11-hydroxy (23-30) derivatives of harmine (1). All compounds were fully characterized.

\section{Results and Discussion}

The solid phase reaction of harmine (1) with different acylating agents (acetic anhydride, propionyl chloride, butyric anhydride, iso-butyric anhydride, valeryl chloride, hexanoyl chloride, heptanoyl chloride and capryloyl chloride) in the presence of $\mathrm{AlCl}_{3}$ yielded mono acyl derivatives without demethylation as well as mono and diacyl derivatives with demethylation. The acylation took place at the carbons ortho to the methoxy group (C-10 acylated products $2-9)$ and (C-12 acylated products 10- 
17). Acylation with demethylation of the $\mathrm{C}-11 \mathrm{OCH}_{3}$ group yielded both monoacyl (C-10-acyl,11hydroxy 18-22) and diacyl (C-10,12-diacyl,11-hydroxy 23-30) products.<smiles>COc1ccc2c(c1)[nH]c1c(C)nccc12</smiles>

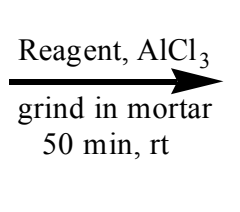<smiles>[R]c1cc2c([nH]c3c(C)nccc32)c([R3])c1[R]</smiles>

Table 1. Friedel-Crafts acylation of $\mathbf{1}$ under solvent-free conditions.

\begin{tabular}{|c|c|c|c|c|c|c|}
\hline Entry & Reagents & Products & $\mathbf{R}_{1}$ & $\mathbf{R}_{\mathbf{2}}$ & $\mathbf{R}_{\mathbf{3}}$ & Yield $^{\mathrm{a}}(\%)$ \\
\hline \multirow[t]{3}{*}{$\mathbf{A}$} & $\left(\mathrm{CH}_{3} \mathrm{CO}\right)_{2} \mathrm{O}$ & 2 & ${ }^{1} \mathrm{CO}-{ }^{2} \mathrm{CH}_{3}$ & $\mathrm{OCH}_{3}$ & $\mathrm{H}$ & 14.4 \\
\hline & & 10 & $\mathrm{H}$ & $\mathrm{OCH}_{3}$ & ${ }^{1} \mathrm{CO}-{ }^{2} \mathrm{CH}_{3}$ & 12.5 \\
\hline & & 23 & ${ }^{1} \mathrm{CO}_{-}{ }^{\prime} \mathrm{CH}_{3}$ & $\mathrm{OH}$ & ${ }^{1 "} \mathrm{CO}_{-}{ }^{2 "} \mathrm{CH}_{3}$ & 12.7 \\
\hline \multirow[t]{4}{*}{ B } & $\mathrm{CH}_{3} \mathrm{CH}_{2} \mathrm{COCl}$ & 3 & ${ }^{1} \mathrm{CO}_{-}{ }^{2} \mathrm{CH}_{2}{ }^{-{ }^{\prime}} \mathrm{CH}_{3}$ & $\mathrm{OCH}_{3}$ & $\mathrm{H}$ & 33.6 \\
\hline & & 11 & $\mathrm{H}$ & $\mathrm{OCH}_{3}$ & ${ }^{1} \mathrm{CO}_{-}{ }^{\prime} \mathrm{CH}_{2}{ }^{3}{ }^{\prime} \mathrm{CH}_{3}$ & 12.7 \\
\hline & & 18 & ${ }^{1} \mathrm{CO}_{-}{ }^{\prime} \mathrm{CH}_{2}-{ }^{3} \mathrm{CH}_{3}$ & $\mathrm{OH}$ & $\mathrm{H}$ & 12.8 \\
\hline & & 24 & ${ }^{1} \mathrm{CO}-{ }^{2} \mathrm{CH}_{2}{ }^{3}{ }^{\prime} \mathrm{CH}_{3}$ & $\mathrm{OH}$ & ${ }^{1 "} \mathrm{CO}-{ }^{2 "} \mathrm{CH}_{2}{ }^{3}{ }^{3 "} \mathrm{CH}_{3}$ & 14.6 \\
\hline \multirow[t]{4}{*}{$\mathbf{C}$} & $\left(\mathrm{CH}_{3}-\mathrm{CH}_{2}-\mathrm{CH}_{2}-\mathrm{CO}\right)_{2} \mathrm{O}$ & 4 & ${ }^{1} \mathrm{CO}-\left(\mathrm{CH}_{2}\right)_{2}{ }^{-{ }^{\prime}} \mathrm{CH}_{3}$ & $\mathrm{OCH}_{3}$ & $\mathrm{H}$ & 32.1 \\
\hline & & 12 & $\mathrm{H}$ & $\mathrm{OCH}_{3}$ & ${ }^{1} \mathrm{CO}-\left(\mathrm{CH}_{2}\right)_{2}{ }^{-{ }^{4}} \mathrm{CH}_{3}$ & 12.5 \\
\hline & & 19 & ${ }^{1} \mathrm{CO}-\left(\mathrm{CH}_{2}\right)_{2}{ }^{4}{ }^{4} \mathrm{CH}_{3}$ & $\mathrm{OH}$ & $\mathrm{H}$ & 7.0 \\
\hline & & 25 & ${ }^{1} \mathrm{CO}-\left(\mathrm{CH}_{2}\right)_{2}{ }^{-1}{ }^{4} \mathrm{CH}_{3}$ & $\mathrm{OH}$ & ${ }^{1 "} \mathrm{CO}-\left(\mathrm{CH}_{2}\right)_{2}{ }^{4}{ }^{4 "} \mathrm{CH}_{3}$ & 9.7 \\
\hline \multirow[t]{4}{*}{ D } & $\left(\left(\mathrm{CH}_{3}\right)_{2} \mathrm{CH}-\mathrm{CO}\right)_{2} \mathrm{O}$ & 5 & ${ }^{1} \mathrm{CO}-{ }^{2} \mathrm{CH}\left(\mathrm{CH}_{3}\right)_{2}$ & $\mathrm{OCH}_{3}$ & $\mathrm{H}$ & 20.7 \\
\hline & & 13 & $\mathrm{H}$ & $\mathrm{OCH}_{3}$ & ${ }^{1} \mathrm{CO}-{ }^{2} \mathrm{CH}\left(\mathrm{CH}_{3}\right)_{2}$ & 7.9 \\
\hline & & 20 & ${ }^{1} \mathrm{CO}-{ }^{2} \mathrm{CH}\left(\mathrm{CH}_{3}\right)_{2}$ & $\mathrm{OH}$ & $\mathrm{H}$ & 20.2 \\
\hline & & 26 & ${ }^{1} \mathrm{CO}-{ }^{2} \mathrm{CH}\left(\mathrm{CH}_{3}\right)_{2}$ & $\mathrm{OH}$ & ${ }^{1 "} \mathrm{CO}_{-}{ }^{2 "} \mathrm{CH}\left(\mathrm{CH}_{3}\right)_{2}$ & 7.9 \\
\hline \multirow[t]{3}{*}{$\mathbf{E}$} & $\mathrm{CH}_{3}-\left(\mathrm{CH}_{2}\right)_{3}-\mathrm{CO}-\mathrm{Cl}$ & 6 & ${ }^{1} \mathrm{CO}-\left(\mathrm{CH}_{2}\right)_{3}{ }^{-}{ }^{5} \mathrm{CH}_{3}$ & $\mathrm{OCH}_{3}$ & $\mathrm{H}$ & 18.8 \\
\hline & & 14 & $\mathrm{H}$ & $\mathrm{OCH}_{3}$ & ${ }^{1} \mathrm{CO}-\left(\mathrm{CH}_{2}\right)_{3}{ }^{-5} \mathrm{CH}_{3}$ & 10.1 \\
\hline & & 27 & ${ }^{1} \mathrm{CO}-\left(\mathrm{CH}_{2}\right)_{3}{ }^{-}{ }^{5} \mathrm{CH}_{3}$ & $\mathrm{OH}$ & ${ }^{1 "} \mathrm{CO}-\left(\mathrm{CH}_{2}\right)_{3}{ }^{-5} \mathrm{CH}_{3}$ & 14.8 \\
\hline \multirow[t]{4}{*}{$\mathbf{F}$} & $\mathrm{CH}_{3}-\left(\mathrm{CH}_{2}\right)_{4}-\mathrm{CO}-\mathrm{Cl}$ & 7 & ${ }^{1} \mathrm{CO}-\left(\mathrm{CH}_{2}\right)_{4}{ }^{-}{ }^{\prime} \mathrm{CH}_{3}$ & $\mathrm{OCH}_{3}$ & $\mathrm{H}$ & 20.0 \\
\hline & & 15 & $\mathrm{H}$ & $\mathrm{OCH}_{3}$ & ${ }^{1} \mathrm{CO}-\left(\mathrm{CH}_{2}\right)_{4}{ }^{-{ }^{6}} \mathrm{CH}_{3}$ & 12.6 \\
\hline & & 21 & ${ }^{1} \mathrm{CO}-\left(\mathrm{CH}_{2}\right)_{4}-{ }^{-}{ }^{\prime} \mathrm{CH}_{3}$ & $\mathrm{OH}$ & $\mathrm{H}$ & 10.2 \\
\hline & & 28 & ${ }^{1} \mathrm{CO}-\left(\mathrm{CH}_{2}\right)_{4}{ }^{-}{ }^{6} \mathrm{CH}_{3}$ & $\mathrm{OH}$ & ${ }^{1 "} \mathrm{CO}-\left(\mathrm{CH}_{2}\right)_{4}{ }^{-6}{ }^{6} \mathrm{CH}_{3}$ & 13.5 \\
\hline \multirow[t]{4}{*}{$\mathbf{G}$} & $\mathrm{CH}_{3}-\left(\mathrm{CH}_{2}\right)_{5}-\mathrm{CO}-\mathrm{Cl}$ & 8 & ${ }^{1} \mathrm{CO}-\left(\mathrm{CH}_{2}\right)_{5}{ }^{-}{ }^{7} \mathrm{CH}_{3}$ & $\mathrm{OCH}_{3}$ & $\mathrm{H}$ & 26.8 \\
\hline & & 16 & $\mathrm{H}$ & $\mathrm{OCH}_{3}$ & ${ }^{1} \mathrm{CO}-\left(\mathrm{CH}_{2}\right)_{5}{ }^{-{ }^{7}} \mathrm{CH}_{3}$ & 11.8 \\
\hline & & 22 & ${ }^{1} \mathrm{CO}-\left(\mathrm{CH}_{2}\right)_{5}{ }^{-{ }^{\prime}} \mathrm{CH}_{3}$ & $\mathrm{OH}$ & $\mathrm{H}$ & 5.9 \\
\hline & & 29 & ${ }^{1} \mathrm{CO}-\left(\mathrm{CH}_{2}\right)_{5^{-}}{ }^{7} \mathrm{CH}_{3}$ & $\mathrm{OH}$ & ${ }^{1 "} \mathrm{CO}-\left(\mathrm{CH}_{2}\right)_{5}{ }^{-7 "} \mathrm{CH}_{3}$ & 14.1 \\
\hline \multirow[t]{3}{*}{$\mathbf{H}$} & $\mathrm{CH}_{3}-\left(\mathrm{CH}_{2}\right)_{6}-\mathrm{CO}-\mathrm{Cl}$ & 9 & ${ }^{1} \mathrm{CO}-\left(\mathrm{CH}_{2}\right)_{6}{ }^{-}{ }^{8} \mathrm{CH}_{3}$ & $\mathrm{OCH}_{3}$ & $\mathrm{H}$ & 31.1 \\
\hline & & 17 & $\mathrm{H}$ & $\mathrm{OCH}_{3}$ & ${ }^{1} \mathrm{CO}-\left(\mathrm{CH}_{2}\right)_{6}{ }^{8}{ }^{8} \mathrm{CH}_{3}$ & 11.5 \\
\hline & & 30 & ${ }^{1} \mathrm{CO}-\left(\mathrm{CH}_{2}\right)_{6}{ }^{-{ }^{\prime}} \mathrm{CH}_{3}$ & $\mathrm{OH}$ & ${ }^{1 "} \mathrm{CO}-\left(\mathrm{CH}_{2}\right)_{6}{ }^{-{ }^{8}{ }^{11} \mathrm{CH}_{3}}$ & 12.7 \\
\hline
\end{tabular}

${ }^{a}$ Isolated yields of the products. 
These reaction products were separated by silica gel column chromatography. It was noted that $10-$ acyl derivatives were obtained as the major products. The remaining derivatives were also obtained in acceptable yields (Table 1). No 10-acyl-11-hydroxy derivatives could be isolated in the present work from the products of the reaction with acetic anhydride (entry A), valeryl chloride (entry E) and capryloyl chloride (entry H). All these harmine derivatives are reported for the first time. The 10,12diacyl-11-hydroxy products may be formed from 10-acyl-11-hydroxy compounds through direct Friedel-Crafts acylation at C-12, or may be obtained after esterification of phenolic $\mathrm{OH}$, through Fries rearrangement in the presence of $\mathrm{AlCl}_{3}$ [15]. The demethylation of 11- $\mathrm{OCH}_{3}$ in the presence of Lewis acid $\left(\mathrm{AlCl}_{3}\right)$ may be due to the electron withdrawing effect of the 10-acyl group and the pyridine ring of harmine. The resulting phenolic $\mathrm{OH}$ facilitates the introduction of second acyl group in the benzene ring and considerable quantities of 10,12-diacyl-11-hydroxy derivatives of harmine were obtained under these solvent-free conditions. Earlier it has been reported that phenols do not react satisfactorily with Friedel-Crafts reagent in solvents because of their reaction with Lewis acids $\left(\mathrm{Ar}-\mathrm{OH}+\mathrm{AlCl}_{3} \rightarrow\right.$ $\left.\mathrm{Ar}-\mathrm{O}-\mathrm{AlCl}_{2}+\mathrm{HCl}\right)$ resulting in compounds which are usually only sparingly soluble in the reaction medium and hence slow down the reactions [16]. It is important to note that under the same reaction conditions Ghiaci and Asghari [17] reported $O$-acylated phenols but in the present studies no $O$ acylated product was obtained. Further, under these reaction conditions no acylation of the pyridine ring of 1 occurred.

Figure 1. Significant $\mathrm{HMBC}\left({ }^{1} \mathrm{H} \rightarrow{ }^{13} \mathrm{C}\right)$ interactions of 10,12-dipropionyl-11-hydroxy (24) analogue of $\mathbf{1 .}$

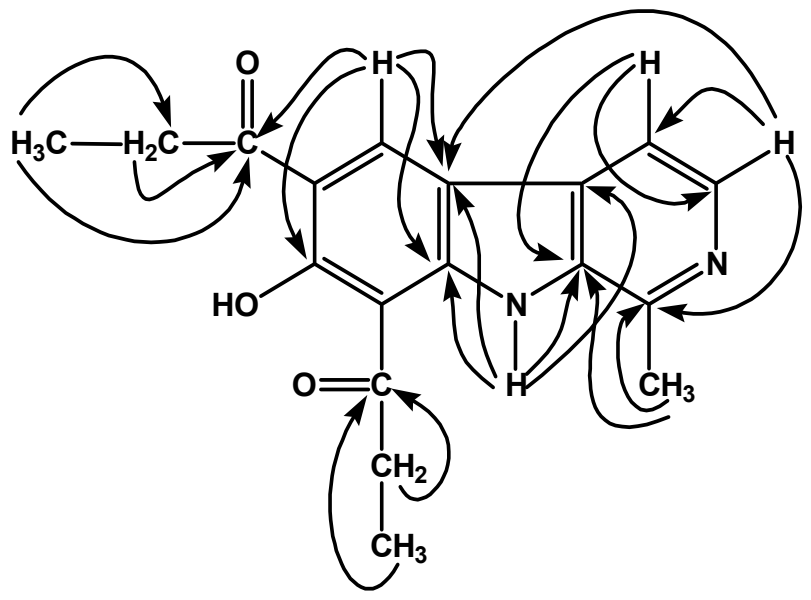

The derivatives have been characterized by spectral studies, including IR, UV, EIMS, HREIMS, 1D $\left({ }^{1} \mathrm{H}-\mathrm{NMR}\right.$ and ${ }^{13} \mathrm{C}-\mathrm{NMR}$; Broad Band decoupled, DEPT), and 2D-NMR (COSY-45, TOCSY, HMQC and HMBC) (See Experimental, Tables 2 and 3) and comparison of the spectral data with reported values of similar compounds [13, 18-19]. For the 10-acyl analogues (see compound 3 in Table 2), the characteristic $\mathrm{H}-9$ and $\mathrm{H}-12$ peaks appeared in the ${ }^{1} \mathrm{H}-\mathrm{NMR}$ spectra as one-proton singlets at $\sim \delta 8.5$ and 7.1, respectively, confirming the substitution at C-10. The ${ }^{1} \mathrm{H}-\mathrm{NMR}$ spectra of 12-acyl analogues (compound 11) characteristically showed two sets of one-proton doublets at $\sim \delta 8.2$ and 6.9, assigned to $\mathrm{H}-9$ and $\mathrm{H}-10$ respectively, showing the substitution on $\mathrm{C}-12$. For 10-acyl demethylated analogues (compound 18), the characteristic peaks of H-9 and H-12 appeared as oneproton singlets at $\sim \delta 8.8$ and 6.9 , respectively, whereas for 10,12-diacyl demethylated analogues 
(compound 24), the characteristic H-9 peak appeared as a one-proton singlet at $\sim \delta 8.7$ and a peak for chelated phenolic $\mathrm{OH}$ appeared at $\sim \delta 14$ in $\mathrm{CDCl}_{3}$ in the ${ }^{1} \mathrm{H}-\mathrm{NMR}$ spectra. The demethylation was also confirmed from their high resolution mass spectral data (See Experimental). The ${ }^{13} \mathrm{C}-\mathrm{NMR}$ signals of quaternary carbons were particularly assigned on the basis of HMBC connectivities observed for these carbons with various protons. The typical HMBC connectivities of various protons with different carbons are also shown in Figure 1 for compound 24.

\section{Conclusions}

In this study new benzene ring substituted and demethylated derivatives of harmine were obtained for the first time in one-pot using acyl halides/acid anhydride and $\mathrm{AlCl}_{3}$ under solvent-free conditions at room temperature after short reaction times. The derivatives obtained in these studies are being screened for potential biological activities such as anti-cancer, anti-bacterial, anti-fungal, CNS and anti-hypertensive properties. The results will be reported in due course. Further the $\beta$-carboline alkaloids possessing acyl substituents on $\mathrm{C}-10$ and $\mathrm{C}-12$ are rare in nature, hence the spectral data presented in this communication should be a useful contribution to the structural elucidation of related natural products.

\section{Experimental}

\section{General}

Melting points were determined using a Buchi-535 melting point apparatus and are uncorrected. Ultraviolet spectra were measured on a Hitachi-3200 spectrophotometer. Infrared spectra were recorded on a Bruker VECTOR 22 spectrophotometer. The ${ }^{1} \mathrm{H}$ - and ${ }^{13} \mathrm{C}$-NMR spectra were recorded at 400 and $100 \mathrm{MHz}$, respectively, on a Bruker Avance 400 spectrometer. Mass spectra were run on a Jeol JMS-HX110 (high resolution, E.I. probe, $70 \mathrm{eV}$ ) and a Varian MAT-312 (low resolution, E.I. probe, $70 \mathrm{eV}$ ) instrument.

\section{Solvent-Free Friedel-Crafts Acylation: General Procedure}

A mixture of harmine $(1,100 \mathrm{mg})$, acylating agents $(3.5 \mathrm{~mL})$ and anhydrous $\mathrm{AlCl}_{3}(400 \mathrm{mg})$ was thoroughly ground in an agate mortar with a pestle for $50 \mathrm{~min}$ in a fume cupboard. The reaction mixture was then kept at room temperature for 1 hour and poured into crushed ice, made basic with $30 \%$ aqueous $\mathrm{NH}_{4} \mathrm{OH}$ and extracted with ethyl acetate. The ethyl acetate layer gave a solid mass after washing, drying $\left(\mathrm{Na}_{2} \mathrm{SO}_{4}\right)$ and removal of solvents under reduced pressure. Further workup using column chromatography (silica gel; Merck 9385; $\mathrm{CHCl}_{3}-\mathrm{MeOH}$, in increasing order of polarity from 9.95:0.05 to 9.5:0.5) afforded 10,12-diacyl,11-hydroxy (23-30; TLC data: $\mathrm{R}_{\mathrm{f}} \sim$ 0.90, 9.5:0.5 $\mathrm{CHCl}_{3^{-}}$ $\mathrm{MeOH}), 12$-acyl (10-17; TLC data: $\mathrm{R}_{\mathrm{f}} \sim 0.86$, 9.5:0.5 $\left.\mathrm{CHCl}_{3}-\mathrm{MeOH}\right), 10$-acyl,11-hydroxy (18-22; TLC data: $\left.\mathrm{R}_{\mathrm{f}} \sim 0.70,9.5: 0.5 \mathrm{CHCl}_{3}-\mathrm{MeOH}\right)$ and 10-acyl (2-9; TLC data: $\mathrm{R}_{\mathrm{f}} \sim$ 0.61, 9.5:0.5 $\mathrm{CHCl}_{3}-$ $\mathrm{MeOH}$ ) derivatives, respectively, as colorless crystalline solids, in moderate yields (Table 1). 
10-Acetyl-11-methoxy-3-methyl- $\beta$-carboline (2). $17.3 \mathrm{mg} ; \mathrm{mp}: 265-266{ }^{\circ} \mathrm{C} ; \mathrm{IR}\left(\mathrm{CHCl}_{3}\right) v_{\max } \mathrm{cm}^{-1}$ : 3263.2 (indole N-H) 2962.6, $2847.8(\mathrm{C}-\mathrm{H}), 1656.4$ (ketone $\mathrm{C}=\mathrm{O}$ ), 1606.1, 1524.2, 1457.0 (aromatic $\mathrm{C}=\mathrm{C}), 1162.3(\mathrm{C}-\mathrm{O})$; UV (MeOH) $\lambda_{\max } \mathrm{nm}: 330.1,270.1,229.7 ;{ }^{1} \mathrm{H}-\mathrm{NMR}\left(\mathrm{CD}_{3} \mathrm{OD}\right): \delta 8.18(1 \mathrm{H}, \mathrm{d}, J$ $=5.7 \mathrm{~Hz}, \mathrm{H}-5), 8.05(1 \mathrm{H}, \mathrm{d}, J=5.7 \mathrm{~Hz}, \mathrm{H}-6), 8.46(1 \mathrm{H}, \mathrm{s}, \mathrm{H}-9), 7.14(1 \mathrm{H}, \mathrm{s}, \mathrm{H}-12), 2.86(3 \mathrm{H}, \mathrm{s}, \mathrm{H}-$ 14), $2.98\left(3 \mathrm{H}, \mathrm{s}, \mathrm{H}-2^{\prime}\right), 4.01\left(3 \mathrm{H}, \mathrm{s}, \mathrm{OCH}_{3}\right) ;{ }^{13} \mathrm{C}-\mathrm{NMR}\left(\mathrm{CD}_{3} \mathrm{OD}\right): \delta 136.5(\mathrm{C}-2), 142.1(\mathrm{C}-3), 139.3(\mathrm{C}-$ 5), 113.2 (C-6), 116.0 (C-7), 130.8 (C-8), 125.9 (C-9), 123.9 (C-10), 161.6 (C-11), 94.5 (C-12), 146.6 (C-13), 19.6 (C-14), $204.6\left(\mathrm{C}-1^{\prime}\right), 32.4\left(\mathrm{C}-2^{\prime}\right), 56.8\left(\mathrm{OCH}_{3}\right)$; HREIMS $(m / z): 254.1048\left[\mathrm{C}_{15} \mathrm{H}_{14} \mathrm{~N}_{2} \mathrm{O}_{2}\right.$ calcd. 254.1055]; EIMS m/z (\%): 254 [M+] (100), 239 (94), 224 (36), 211 (4), 196 (28).

10-Propionyl-11-methoxy-3-methyl- $\beta$-carboline (3). $39.2 \mathrm{mg}$; mp: $268-269{ }^{\circ} \mathrm{C}$; IR $\left(\mathrm{CHCl}_{3}\right) v_{\max } \mathrm{cm}^{-1}$ : 3264.2 (indole $\mathrm{N}-\mathrm{H}$ ), 2963.2, $2848.6(\mathrm{C}-\mathrm{H}), 1656.1$ (ketone $\mathrm{C}=\mathrm{O}$ ), 1604.1, 1525.1, 1453.2 (aromatic $\mathrm{C}=\mathrm{C}), 1166.3$ (C-O); UV (MeOH) $\lambda_{\max } \mathrm{nm}: 329.6,269.4,230.2 ;{ }^{1} \mathrm{H}-\mathrm{NMR}\left(\mathrm{CD}_{3} \mathrm{OD}\right)$ : Table $2 ;{ }^{13} \mathrm{C}-$ NMR (CD 3 OD): Table 3; HREIMS ( $m / z)$ : $268.1202\left[\mathrm{C}_{16} \mathrm{H}_{16} \mathrm{~N}_{2} \mathrm{O}_{2}\right.$ calcd. 268.1212]; EIMS $\mathrm{m} / z(\%)$ : $268\left[\mathrm{M}^{+}\right](60), 253$ (10), 239 (100), 224 (40), 211 (3), 196 (32).

10-Butyryl-11-methoxy-3-methyl- $\beta$-carboline (4). $42.7 \mathrm{mg}$; mp: $269-270{ }^{\circ} \mathrm{C}$; IR $\left(\mathrm{CHCl}_{3}\right) v_{\max } \mathrm{cm}^{-1}$ : 3268.2 (indole $\mathrm{N}-\mathrm{H}$ ), 2927.1, $2854.2(\mathrm{C}-\mathrm{H}), 1657.1$ (ketone $\mathrm{C}=\mathrm{O}), 1608.3,1523.9,1412.5$ (aromatic $\mathrm{C}=\mathrm{C}), 1152.1(\mathrm{C}-\mathrm{O})$; UV (MeOH) $\lambda_{\max } \mathrm{nm}: 329.8,269.8,229.5 ;{ }^{1} \mathrm{H}-\mathrm{NMR}\left(\mathrm{CD}_{3} \mathrm{OD}\right): \delta 8.18(1 \mathrm{H}, \mathrm{d}, J$ $=5.4 \mathrm{~Hz}, \mathrm{H}-5), 8.04(1 \mathrm{H}, \mathrm{d}, J=5.4 \mathrm{~Hz}, \mathrm{H}-6), 8.48(1 \mathrm{H}, \mathrm{s}, \mathrm{H}-9), 7.15(1 \mathrm{H}, \mathrm{s}, \mathrm{H}-12), 2.88(3 \mathrm{H}, \mathrm{s}, \mathrm{H}-$ 14), 3.03 (2H, t, $\left.J=7.3 \mathrm{~Hz}, \mathrm{H}-2^{\prime}\right), 1.72$ (2H, sextet, $\left.J=7.3 \mathrm{~Hz}, \mathrm{H}-3^{\prime}\right), 0.99$ (3H, t, $\left.J=7.3 \mathrm{~Hz}, \mathrm{H}-4^{\prime}\right)$, $4.03\left(3 \mathrm{H}, \mathrm{s}, \mathrm{OCH}_{3}\right) ;{ }^{13} \mathrm{C}-\mathrm{NMR}\left(\mathrm{CD}_{3} \mathrm{OD}\right): \delta 136.3(\mathrm{C}-2), 142.5(\mathrm{C}-3), 139.7(\mathrm{C}-5), 113.6(\mathrm{C}-6), 116.1$ (C-7), 130.9 (C-8), 125.7 (C-9), 123.8 (C-10), 161.5 (C-11), 94.6 (C-12), 146.4 (C-13), 19.0 (C-14), $204.3\left(\mathrm{C}-1^{\prime}\right), 46.0\left(\mathrm{C}-2^{\prime}\right), 18.6\left(\mathrm{C}-3^{\prime}\right), 14.2\left(\mathrm{C}-4^{\prime}\right), 56.8\left(\mathrm{OCH}_{3}\right) ; \operatorname{HREIMS}(\mathrm{m} / \mathrm{z}): 282.1360$ [ $\mathrm{C}_{17} \mathrm{H}_{18} \mathrm{~N}_{2} \mathrm{O}_{2}$ calcd. 282.1368]; EIMS m/z (\%): $282\left[\mathrm{M}^{+}\right]$(30), 267 (6), 239 (100), 224 (10), 211 (2), 196 (28).

10-Isobutyryl-11-methoxy-3-methyl- $\beta$-carboline (5). $27.5 \mathrm{mg} ; \mathrm{mp}: 273-274{ }^{\circ} \mathrm{C}$; IR $\left(\mathrm{CHCl}_{3}\right) v_{\max } \mathrm{cm}^{-1}$ : 3252.2 (indole $\mathrm{N}-\mathrm{H}$ ), 2947.0, $2853.1(\mathrm{C}-\mathrm{H}), 1656.2$ (ketone $\mathrm{C}=\mathrm{O}$ ), 1607.5, 1524.7, 1456.5 (aromatic $\mathrm{C}=\mathrm{C}), 1152.2(\mathrm{C}-\mathrm{O})$; UV (MeOH) $\lambda_{\max } \mathrm{nm}: 330.0,269.7,229.5 ;{ }^{1} \mathrm{H}-\mathrm{NMR}\left(\mathrm{CD}_{3} \mathrm{OD}\right): \delta 8.20(1 \mathrm{H}, \mathrm{d}, J$ $=5.5 \mathrm{~Hz}, \mathrm{H}-5), 8.04(1 \mathrm{H}, \mathrm{d}, J=5.5 \mathrm{~Hz}, \mathrm{H}-6), 8.47(1 \mathrm{H}, \mathrm{s}, \mathrm{H}-9), 7.14(1 \mathrm{H}, \mathrm{s}, \mathrm{H}-12), 2.86(3 \mathrm{H}, \mathrm{s}, \mathrm{H}-$ 14), $3.60\left(1 \mathrm{H}\right.$, septet, $\left.J=6.8 \mathrm{~Hz}, \mathrm{H}-2^{\prime}\right), 1.13\left(6 \mathrm{H}, \mathrm{d}, J=6.8 \mathrm{~Hz}, \mathrm{H}-3^{\prime}\right.$ and $\left.\mathrm{H}-4^{\prime}\right), 4.02\left(3 \mathrm{H}, \mathrm{s}, \mathrm{OCH}_{3}\right)$; ${ }^{13} \mathrm{C}-\mathrm{NMR}\left(\mathrm{CD}_{3} \mathrm{OD}\right): \delta 136.4$ (C-2), 142.2 (C-3), 139.2 (C-5), 113.5 (C-6), 116.3 (C-7), 130.5 (C-8), 125.8 (C-9), 124.1 (C-10), 161.4 (C-11), 94.5 (C-12), 146.4 (C-13), 19.2 (C-14), 208.4 (C-1'), 40.1 (C$\left.2^{\prime}\right), 18.8\left(\mathrm{C}-3^{\prime}\right.$ and $\left.\mathrm{C}-4^{\prime}\right), 56.5\left(\mathrm{OCH}_{3}\right)$; HREIMS $(\mathrm{m} / \mathrm{z}): 282.1357\left[\mathrm{C}_{17} \mathrm{H}_{18} \mathrm{~N}_{2} \mathrm{O}_{2}\right.$ calcd. 282.1368]; EIMS $m / z(\%): 282\left[\mathrm{M}^{+}\right](92), 239$ (100), $224(6), 211(8), 196(21)$.

10-Valeryl-11-hydroxy-3-methyl- $\beta$-carboline (6). $26.3 \mathrm{mg}$; mp: 272-273 ${ }^{\circ} \mathrm{C}$; IR $\left(\mathrm{CHCl}_{3}\right) v_{\max } \mathrm{cm}^{-1}$ : 3252.6 (indole $\mathrm{N}-\mathrm{H}$ ), 2928.0, $2860.2(\mathrm{C}-\mathrm{H}), 1655.8$ (ketone $\mathrm{C}=\mathrm{O}$ ), 1601.6, 1525.4, 1423.1 (aromatic $\mathrm{C}=\mathrm{C}), 1152.8(\mathrm{C}-\mathrm{O})$; UV (MeOH) $\lambda_{\max } \mathrm{nm}: 330.2,269.8,229.6 ;{ }^{1} \mathrm{H}-\mathrm{NMR}\left(\mathrm{CD}_{3} \mathrm{OD}\right): \delta 8.18(1 \mathrm{H}, \mathrm{d}, J$ $=5.8 \mathrm{~Hz}, \mathrm{H}-5), 8.08(1 \mathrm{H}, \mathrm{d}, J=5.8 \mathrm{~Hz}, \mathrm{H}-6), 8.45(1 \mathrm{H}, \mathrm{s}, \mathrm{H}-9), 7.12(1 \mathrm{H}, \mathrm{s}, \mathrm{H}-12), 2.87(3 \mathrm{H}, \mathrm{s}, \mathrm{H}-$ 14), $3.02\left(2 \mathrm{H}, \mathrm{t}, J=7.3 \mathrm{~Hz}, \mathrm{H}-2^{\prime}\right), 1.66\left(2 \mathrm{H}\right.$, quintet, $\left.J=7.3 \mathrm{~Hz}, \mathrm{H}-3^{\prime}\right), 1.40$ (2H, sextet, $J=7.3 \mathrm{~Hz}$, H-4'), $0.94\left(3 \mathrm{H}, \mathrm{t}, J=7.3 \mathrm{~Hz}, \mathrm{H}-5^{\prime}\right), 4.03\left(3 \mathrm{H}, \mathrm{s}, \mathrm{OCH}_{3}\right) ;{ }^{13} \mathrm{C}-\mathrm{NMR}\left(\mathrm{CD}_{3} \mathrm{OD}\right): \delta 136.5(\mathrm{C}-2), 142.6$ 
(C-3), 139.0 (C-5), 113.8 (C-6), 116.0 (C-7), 130.7 (C-8), 126.5 (C-9), 124.2 (C-10), 161.5 (C-11), 94.7 (C-12), 146.7 (C-13), 19.4 (C-14), 204.5 (C-1'), 44.2 (C-2'), 28.1 (C-3'), 23.5 (C-4'), 14.3 (C-5'), 56.6 $\left(\mathrm{OCH}_{3}\right)$; HREIMS (m/z): 296.1512 [ $\mathrm{C}_{18} \mathrm{H}_{20} \mathrm{~N}_{2} \mathrm{O}_{2}$ calcd. 296.1525]; EIMS m/z (\%): 296 [M $\left.{ }^{+}\right](12)$, 267 (2), 254 (14), 239 (100), 224 (8), 211 (3), 196 (7).

10-Hexanoyl-11-methoxy-3-methyl- $\beta$-carboline (7). $29.3 \mathrm{mg} ; \mathrm{mp}: 274-275{ }^{\circ} \mathrm{C} ; \mathrm{IR}\left(\mathrm{CHCl}_{3}\right) v_{\max } \mathrm{cm}^{-1}$ : 3251.8 (indole $\mathrm{N}-\mathrm{H}$ ), 2925.8, $2856.4(\mathrm{C}-\mathrm{H}), 1655.2$ (ketone $\mathrm{C}=\mathrm{O}$ ), 1602.3, 1523.3, 1458.1 (aromatic $\mathrm{C}=\mathrm{C}), 1168.1$ (C-O); UV (MeOH) $\lambda_{\max } \mathrm{nm}: 330.1,269.8,231.4 ;{ }^{1} \mathrm{H}-\mathrm{NMR}\left(\mathrm{CD}_{3} \mathrm{OD}\right): \delta 8.19(1 \mathrm{H}, \mathrm{d}, J$ $=5.4 \mathrm{~Hz}, \mathrm{H}-5), 8.03(1 \mathrm{H}, \mathrm{d}, J=5.4 \mathrm{~Hz}, \mathrm{H}-6), 8.45(1 \mathrm{H}, \mathrm{s}, \mathrm{H}-9), 7.12(1 \mathrm{H}, \mathrm{s}, \mathrm{H}-12), 2.85$ (3H, s, H14), $3.02\left(2 \mathrm{H}, \mathrm{t}, J=7.4 \mathrm{~Hz}, \mathrm{H}-2^{\prime}\right), 1.68$ (2H, quintet, $\left.J=7.4 \mathrm{~Hz}, \mathrm{H}-3^{\prime}\right), 1.36$ (4H, m, H-4' and H-5'), $0.91\left(3 \mathrm{H}, \mathrm{t}, J=7.4 \mathrm{~Hz}, \mathrm{H}-6^{\prime}\right), 4.01\left(3 \mathrm{H}, \mathrm{s}, \mathrm{OCH}_{3}\right) ;{ }^{13} \mathrm{C}-\mathrm{NMR}\left(\mathrm{CD}_{3} \mathrm{OD}\right): \delta 137.1(\mathrm{C}-2), 142.7(\mathrm{C}-3)$, 139.1 (C-5), 113.7 (C-6), 116.2 (C-7), 130.6 (C-8), 125.8 (C-9), 124.0 (C-10), 161.6 (C-11), 94.5 (C12), 146.5 (C-13), 19.6 (C-14), 204.8 (C-1'), 44.5 (C-2'), 25.7 (C-3'), 32.8 (C-4'), 23.6 (C-5'), 14.3 (C-

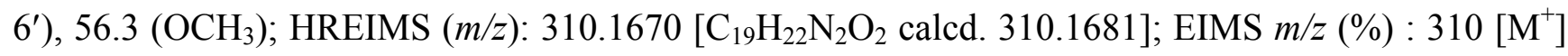
(8), 267 (4), 254 (18), 239 (100), 224 (8), 211 (2), 196 (7).

10-Heptanoyl-11-methoxy-3-methyl- $\beta$-carboline (8). $40.9 \mathrm{mg}$; mp: 277-278 ${ }^{\circ} \mathrm{C}$; IR $\left(\mathrm{CHCl}_{3}\right) v_{\max } \mathrm{cm}^{-1}$ : 3255.6 (indole N-H), 2923.5, $2858.1(\mathrm{C}-\mathrm{H}), 1656.8$ (ketone $\mathrm{C}=\mathrm{O}$ ), 1608.2, 1526.1, 1453.1 (aromatic $\mathrm{C}=\mathrm{C}), 1150.2(\mathrm{C}-\mathrm{O})$; UV (MeOH) $\lambda_{\max } \mathrm{nm}: 330.2,269.3,231.8 ;{ }^{1} \mathrm{H}-\mathrm{NMR}\left(\mathrm{CD}_{3} \mathrm{OD}\right): \delta 8.21(1 \mathrm{H}, \mathrm{d}, J$ $=5.4 \mathrm{~Hz}, \mathrm{H}-5), 8.05(1 \mathrm{H}, \mathrm{d}, J=5.4 \mathrm{~Hz}, \mathrm{H}-6), 8.47(1 \mathrm{H}, \mathrm{s}, \mathrm{H}-9), 7.15(1 \mathrm{H}, \mathrm{s}, \mathrm{H}-12), 2.87$ (3H, s, H14), 2.99 (2H, t, $\left.J=7.2 \mathrm{~Hz}, \mathrm{H}-2^{\prime}\right), 1.66$ (2H, m, H-3'), 1.32 (6H, m, H-4', H-5' and H-6'), 0.90 (3H, t, $J$ $\left.=7.2 \mathrm{~Hz}, \mathrm{H}-7^{\prime}\right), 3.99\left(3 \mathrm{H}, \mathrm{s}, \mathrm{OCH}_{3}\right) ;{ }^{13} \mathrm{C}-\mathrm{NMR}\left(\mathrm{CD}_{3} \mathrm{OD}\right): \delta 136.9(\mathrm{C}-2), 142.5(\mathrm{C}-3), 139.3(\mathrm{C}-5)$, 113.5 (C-6), 116.3 (C-7), 130.6 (C-8), 126.0 (C-9), 124.3 (C-10), 162.0 (C-11), 94.7 (C-12), 146.7 (C13), 19.7 (C-14), 204.1 (C-1'), 44.6 (C-2'), 24.2 (C-3'), 30.3 (C-4'), 32.2 (C-5'), 23.2 (C-6'), 14.6 (C$\left.7^{\prime}\right), 56.6\left(\mathrm{OCH}_{3}\right)$; HREIMS (m/z): $324.1851\left[\mathrm{C}_{20} \mathrm{H}_{24} \mathrm{~N}_{2} \mathrm{O}_{2}\right.$ calcd. 324.1838]; EIMS m/z (\%): 324 [M $\left.{ }^{+}\right]$ (14), 267 (5), 254 (22) 239 (100), 224 (8), 211 (3), 196 (9).

10-Capryloyl-11-methoxy-3-methyl- $\beta$-carboline (9). $49.6 \mathrm{mg} ; \mathrm{mp}: 280-281{ }^{\circ} \mathrm{C}$; IR $\left(\mathrm{CHCl}_{3}\right) v_{\max } \mathrm{cm}^{-1}$ : 3250.2 (indole $\mathrm{N}-\mathrm{H}$ ), 2925.8, $2856.4(\mathrm{C}-\mathrm{H}), 1657.1$ (ketone $\mathrm{C}=\mathrm{O}$ ), 1607.8, 1521.8, 1454.8 (aromatic $\mathrm{C}=\mathrm{C}), 1152.4(\mathrm{C}-\mathrm{O})$; UV (MeOH) $\lambda_{\max } \mathrm{nm}: 330.8,270.1,232.2 ;{ }^{1} \mathrm{H}-\mathrm{NMR}\left(\mathrm{CD}_{3} \mathrm{OD}\right): \delta 8.22(1 \mathrm{H}, \mathrm{d}, J$ $=5.6 \mathrm{~Hz}, \mathrm{H}-5), 8.06(1 \mathrm{H}, \mathrm{d}, J=5.6 \mathrm{~Hz}, \mathrm{H}-6), 8.46(1 \mathrm{H}, \mathrm{s}, \mathrm{H}-9), 7.13(1 \mathrm{H}, \mathrm{s}, \mathrm{H}-12), 2.89$ (3H, s, H14), 3.04 (2H, t, $\left.J=7.3 \mathrm{~Hz}, \mathrm{H}-2^{\prime}\right), 1.69$ (2H, quintet, $\left.J=7.3 \mathrm{~Hz}, \mathrm{H}-3^{\prime}\right), 1.35$ (2H, quintet, $J=7.3 \mathrm{~Hz}$, $\left.\mathrm{H}-4^{\prime}\right), 1.33$ (6H, m, H-5', H-6' and H-7'), $0.88\left(3 \mathrm{H}, \mathrm{t}, J=7.3 \mathrm{~Hz}, \mathrm{H}-8^{\prime}\right), 4.05\left(3 \mathrm{H}, \mathrm{s}, \mathrm{OCH}_{3}\right) ;{ }^{13} \mathrm{C}-\mathrm{NMR}$ $\left(\mathrm{CD}_{3} \mathrm{OD}\right): \delta 136.1$ (C-2), 142.6 (C-3), 139.2 (C-5), 113.8 (C-6), 116.1 (C-7), 130.5 (C-8), 126.1 (C-9), 124.2 (C-10), 161.4 (C-11), 94.8 (C-12), 146.6 (C-13), 19.5 (C-14), 204.5 (C-1'), 43.9 (C-2'), 25.1 (C$\left.3^{\prime}\right)^{*}, 29.8\left(\mathrm{C}-4^{\prime}\right)^{* *}, 29.6\left(\mathrm{C}-5^{\prime}\right)^{* *}, 31.9\left(\mathrm{C}-6^{\prime}\right), 25.4\left(\mathrm{C}-7^{\prime}\right)^{*}, 14.5\left(\mathrm{C}-8^{\prime}\right), 56.7\left(\mathrm{OCH}_{3}\right)\left(^{*}, * *\right.$ values may be interchanged); HREIMS (m/z): $338.1985\left[\mathrm{C}_{21} \mathrm{H}_{26} \mathrm{~N}_{2} \mathrm{O}_{2}\right.$ calcd. 338.1994]; EIMS m/z (\%): 338 $\left[\mathrm{M}^{+}\right]$(6), 267 (5), 254 (21), 239 (100), 224 (10), 211 (8), 196 (6).

12-Acetyl-11-methoxy-3-methyl- $\beta$-carboline (10). $15.0 \mathrm{mg}$; mp: 283-284 ${ }^{\circ} \mathrm{C}$; IR $\left(\mathrm{CHCl}_{3}\right) v_{\max } \mathrm{cm}^{-1}$ : 3388.7 (indole $\mathrm{N}-\mathrm{H}$ ), 2922, $2848.7(\mathrm{C}-\mathrm{H}), 1648.2$ (ketone $\mathrm{C}=\mathrm{O}$ ), 1608.3, 1545.7, 1427.2 (aromatic $\mathrm{C}=\mathrm{C}), 1151.6(\mathrm{C}-\mathrm{O})$; UV $(\mathrm{MeOH}) \lambda_{\max } \mathrm{nm}: 361.8,321.3,269.7,232.5 ;{ }^{1} \mathrm{H}-\mathrm{NMR}\left(\mathrm{CDCl}_{3}\right): \delta 8.35(1 \mathrm{H}$, 
$\mathrm{d}, J=5.3 \mathrm{~Hz}, \mathrm{H}-5), 7.83(1 \mathrm{H}, \mathrm{d}, J=5.3 \mathrm{~Hz}, \mathrm{H}-6), 8.23(1 \mathrm{H}, \mathrm{d}, J=8.6 \mathrm{~Hz}, \mathrm{H}-9), 6.99(1 \mathrm{H}, \mathrm{d}, J=8.6$ Hz, H-10), 2.95 (3H, s, H-14), 2.78 (3H, s, H-2'), 4.09 (3H, s, $\left.\mathrm{OCH}_{3}\right), 11.17$ (1H, br.s, indole N-H); ${ }^{13} \mathrm{C}-\mathrm{NMR}\left(\mathrm{CDCl}_{3}\right): \delta 134.2$ (C-2), $137.9(\mathrm{C}-3), 131.2$ (C-5), 113.9 (C-6), $115.9(\mathrm{C}-7), 131.8(\mathrm{C}-8)$, 129.4 (C-9), 107.7 (C-10), 163.8 (C-11), 109.7 (C-12), 145.0 (C-13), 16.2 (C-14), 203.2 (C-1'), 33.6 $\left(\mathrm{C}-2^{\prime}\right), 56.6\left(\mathrm{OCH}_{3}\right)$; HREIMS $(\mathrm{m} / \mathrm{z}): 254.1042\left[\mathrm{C}_{15} \mathrm{H}_{14} \mathrm{~N}_{2} \mathrm{O}_{2}\right.$ calcd. 254.1055]; EIMS $m / z(\%): 254$ $\left[\mathrm{M}^{+}\right](96), 239$ (100), 224 (31), 211 (3), 196 (33).

12-Propionyl-11-methoxy-3-methyl- $\beta$-carboline (11). $15.7 \mathrm{mg}$; mp: $284-285{ }^{\circ} \mathrm{C}$; IR $\left(\mathrm{CHCl}_{3}\right) v_{\max } \mathrm{cm}^{-1}$ : 3388.2 (indole $\mathrm{N}-\mathrm{H}$ ), 2934.1, $2841.2(\mathrm{C}-\mathrm{H}), 1648.7$ (ketone $\mathrm{C}=\mathrm{O}$ ), 1607.2, 1545.7, 1472.1 (aromatic $\mathrm{C}=\mathrm{C}), 1152.4(\mathrm{C}-\mathrm{O})$; UV $(\mathrm{MeOH}) \lambda_{\max } \mathrm{nm}: 361.4$, 321.6, 269.4, 232.5; ${ }^{1} \mathrm{H}-\mathrm{NMR}\left(\mathrm{CD}_{3} \mathrm{OD}\right)$ : Table 2; ${ }^{13} \mathrm{C}-\mathrm{NMR}\left(\mathrm{CD}_{3} \mathrm{OD}\right)$ : Table 3; HREIMS $(\mathrm{m} / \mathrm{z}): 268.1223\left[\mathrm{C}_{16} \mathrm{H}_{16} \mathrm{~N}_{2} \mathrm{O}_{2}\right.$ calcd. 268.1212]; EIMS $\mathrm{m} / \mathrm{z}$ (\%): $268\left[\mathrm{M}^{+}\right](46),$,253 (4), 239 (100), 224 (31), 211 (4), 196 (29).

12-Butyryl-11-methoxy-3-methyl- $\beta$-carboline (12). $10.5 \mathrm{mg} ; \mathrm{mp}: 287-288{ }^{\circ} \mathrm{C}$; IR $\left(\mathrm{CHCl}_{3}\right) v_{\max } \mathrm{cm}^{-1}$ : 3390.1 (indole N-H) 2956.7, $2871.8(\mathrm{C}-\mathrm{H}), 1647.9$ (ketone $\mathrm{C}=\mathrm{O}$ ), 1610.3, 1544.5, 1434.9 (aromatic $\mathrm{C}=\mathrm{C}), 1152.4(\mathrm{C}-\mathrm{O})$; UV (MeOH) $\lambda_{\max } \mathrm{nm}: 361.2,321.4,269.8,232.4 \mathrm{~nm} ;{ }^{1} \mathrm{H}-\mathrm{NMR}\left(\mathrm{CDCl}_{3}\right): \delta 8.33$ $(1 \mathrm{H}, \mathrm{d}, J=5.4 \mathrm{~Hz}, \mathrm{H}-5), 7.81(1 \mathrm{H}, \mathrm{d}, J=5.4 \mathrm{~Hz}, \mathrm{H}-6), 8.19(1 \mathrm{H}, \mathrm{d}, J=8.7 \mathrm{~Hz}, \mathrm{H}-9), 6.95(1 \mathrm{H}, \mathrm{d}, J=$ $8.7 \mathrm{~Hz}, \mathrm{H}-10), 2.98$ (3H, s, H-14), 3.14 (2H, t, $\left.J=7.3 \mathrm{~Hz}, \mathrm{H}-2^{\prime}\right), 1.79$ (2H, sextet, $\left.J=7.3 \mathrm{~Hz}, \mathrm{H}-3^{\prime}\right)$, $1.03\left(3 \mathrm{H}, \mathrm{t}, J=7.3 \mathrm{~Hz}, \mathrm{H}-44^{\prime}\right), 4.06\left(3 \mathrm{H}, \mathrm{s}, \mathrm{OCH}_{3}\right), 11.16\left(1 \mathrm{H}\right.$, br.s, indole N-H); ${ }^{13} \mathrm{C}-\mathrm{NMR}\left(\mathrm{CDCl}_{3}\right): \delta$ 134.4 (C-2), 138.1 (C-3), 131.0 (C-5), 113.9 (C-6), 116.0 (C-7), 131.9 (C-8), 129.3 (C-9), 107.5 (C10), 163.6 (C-11), 109.8 (C-12), 145.0 (C-13), 16.4 (C-14), 203.3 (C-1'), 45.3 (C-2'), 18.0 (C-3'), 14.2 $\left(\mathrm{C}-4^{\prime}\right), 56.9\left(\mathrm{OCH}_{3}\right)$; HREIMS $(\mathrm{m} / \mathrm{z}): 282.1377\left[\mathrm{C}_{17} \mathrm{H}_{18} \mathrm{~N}_{2} \mathrm{O}_{2}\right.$ calcd. 282.1368]; EIMS m/z (\%): 282 $\left[\mathrm{M}^{+}\right]$(32), 267 (8), 239 (100), 224 (17), 211 (3), 196 (28).

12-Isobutyryl-11-methoxy-3-methyl- $\beta$-carboline (13). $16.6 \mathrm{mg}$; mp: $290-291{ }^{\circ} \mathrm{C}$; IR $\left(\mathrm{CHCl}_{3}\right) v_{\max } \mathrm{cm}^{-1}$ : 3384.1 (indole $\mathrm{N}-\mathrm{H}$ ), 2927.2, $2857.2(\mathrm{C}-\mathrm{H}), 1648.9$ (ketone $\mathrm{C}=\mathrm{O}$ ), 1614.2, 1544.7, 1460.6 (aromatic $\mathrm{C}=\mathrm{C}), 1151.3(\mathrm{C}-\mathrm{O})$; UV (MeOH) $\lambda_{\max } \mathrm{nm}: 361.3,321.4,269.4,232.2 ;{ }^{1} \mathrm{H}-\mathrm{NMR}\left(\mathrm{CDCl}_{3}\right): \delta 8.34(1 \mathrm{H}$, d, $J=5.5 \mathrm{~Hz}, \mathrm{H}-5), 7.80(1 \mathrm{H}, \mathrm{d}, J=5.5 \mathrm{~Hz}, \mathrm{H}-6), 8.21(1 \mathrm{H}, \mathrm{d}, J=8.7 \mathrm{~Hz}, \mathrm{H}-9), 6.98$ (1H, d, $J=8.7$ Hz, H-10), 2.96 (3H, s, H-14), 3.88 (1H, septet, $\left.J=6.7 \mathrm{~Hz}, \mathrm{H}-2^{\prime}\right), 1.23\left(6 \mathrm{H}, \mathrm{d}, J=6.7 \mathrm{~Hz}, \mathrm{H}-3^{\prime}\right.$ and H$\left.4^{\prime}\right), 4.08\left(3 \mathrm{H}, \mathrm{s}, \mathrm{OCH}_{3}\right), 11.21(1 \mathrm{H}$, br.s, indole $\mathrm{N}-\mathrm{H}) ;{ }^{13} \mathrm{C}-\mathrm{NMR}\left(\mathrm{CDCl}_{3}\right): \delta 134.2(\mathrm{C}-2), 138.1(\mathrm{C}-3)$, 131.3 (C-5), 113.6 (C-6), 116.2 (C-7), 131.8 (C-8), 129.2 (C-9), 107.5 (C-10), 163.8 (C-11), 109.6 (C12), 145.1 (C-13), 16.1 (C-14), 207.4 (C-1'), 40.7 (C-2'), 18.8 (C-3' and C-4'), $56.8\left(\mathrm{OCH}_{3}\right)$; HREIMS (m/z): $282.1362\left[\mathrm{C}_{17} \mathrm{H}_{18} \mathrm{~N}_{2} \mathrm{O}_{2}\right.$ calcd. 282.1368]; EIMS m/z (\%): 282 [M $\left.{ }^{+}\right]$(94), 239 (100), 224 (8), 211 (6), $196(17)$.

12-Valeryl-11-methoxy-3-methyl- $\beta$-carboline (14). $14.1 \mathrm{mg} ; \mathrm{mp}: 289-290{ }^{\circ} \mathrm{C}$; IR $\left(\mathrm{CHCl}_{3}\right) v_{\max } \mathrm{cm}^{-1}$ : 3385.1 (indole $\mathrm{N}-\mathrm{H}$ ), 2928.1, $2861.5(\mathrm{C}-\mathrm{H}), 1648.8$ (ketone $\mathrm{C}=\mathrm{O}$ ), 1612.5, 1543.9, 1416.2 (aromatic $\mathrm{C}=\mathrm{C}), 1152.2(\mathrm{C}-\mathrm{O})$; UV $(\mathrm{MeOH}) \lambda_{\max } \mathrm{nm}: 361.2,321.8,269.4,232.6 ;{ }^{1} \mathrm{H}-\mathrm{NMR}\left(\mathrm{CDCl}_{3}\right): \delta 8.34(1 \mathrm{H}$, d, $J=5.3 \mathrm{~Hz}, \mathrm{H}-5), 7.84(1 \mathrm{H}, \mathrm{d}, J=5.3 \mathrm{~Hz}, \mathrm{H}-6), 8.25(1 \mathrm{H}, \mathrm{d}, J=8.7 \mathrm{~Hz}, \mathrm{H}-9), 7.03$ (1H, d, $J=8.7$ Hz, H-10), 3.02 (3H, s, H-14), 3.16 (2H, t, $\left.J=7.2 \mathrm{~Hz}, \mathrm{H}-2^{\prime}\right), 1.73$ (2H, quintet, $J=7.2 \mathrm{~Hz}, \mathrm{H}-3^{\prime}$ ), 1.45 $\left(2 \mathrm{H}\right.$, sextet, $\left.J=7.2 \mathrm{~Hz}, \mathrm{H}-4^{\prime}\right), 0.97\left(3 \mathrm{H}, \mathrm{t}, J=7.2 \mathrm{~Hz}, \mathrm{H}-5^{\prime}\right), 4.10\left(3 \mathrm{H}, \mathrm{s}, \mathrm{OCH}_{3}\right), 11.26(1 \mathrm{H}$, br.s, indole N-H); ${ }^{13} \mathrm{C}-\mathrm{NMR}\left(\mathrm{CDCl}_{3}\right): \delta 134.2$ (C-2), 138.4 (C-3), 131.4 (C-5), 113.8 (C-6), 116.0 (C-7), 
132.1 (C-8), 129.4 (C-9), 107.6 (C-10), 163.9 (C-11), 110.1 (C-12), 144.9 (C-13), 16.2 (C-14), 203.2 (C-1'), 45.0 (C-2'), 26.5 (C-3'), 22.6 (C-4'), $14.0\left(\mathrm{C}-5^{\prime}\right), 56.7\left(\mathrm{OCH}_{3}\right)$; HREIMS (m/z): 296.1514 [ $\mathrm{C}_{18} \mathrm{H}_{20} \mathrm{~N}_{2} \mathrm{O}_{2}$ calcd. 296.1525]; EIMS m/z (\%): $296\left[\mathrm{M}^{+}\right]$(86), 267 (39), 254 (52), 239 (100), 224 (19), $211(2), 196(10)$.

12-Hexanoyl-11-methoxy-3-methyl- $\beta$-carboline (15). $18.4 \mathrm{mg}$; mp: 292-293 ${ }^{\circ} \mathrm{C}$; IR $\left(\mathrm{CHCl}_{3}\right) v_{\max } \mathrm{cm}^{-1}$ : 3385.0 (indole N-H), 2924.3, $2854.1(\mathrm{C}-\mathrm{H}), 1648.8$ (ketone $\mathrm{C}=\mathrm{O}$ ), 1614.5, 1546.4, 1460.0 (aromatic $\mathrm{C}=\mathrm{C}), 1151.8(\mathrm{C}-\mathrm{O})$; UV $(\mathrm{MeOH}) \lambda_{\max } \mathrm{nm}: 361.2,321.5,269.3,232.8 ;{ }^{1} \mathrm{H}-\mathrm{NMR}\left(\mathrm{CDCl}_{3}\right): \delta 8.35(1 \mathrm{H}$, $\mathrm{d}, J=5.4 \mathrm{~Hz}, \mathrm{H}-5), 7.86(1 \mathrm{H}, \mathrm{d}, J=5.4 \mathrm{~Hz}, \mathrm{H}-6), 8.20(1 \mathrm{H}, \mathrm{d}, J=8.6 \mathrm{~Hz}, \mathrm{H}-9), 6.95$ (1H, d, $J=8.6$ Hz, H-10), 2.97 (3H, s, H-14), 3.15 (2H, t, $\left.J=7.3 \mathrm{~Hz}, \mathrm{H}-2^{\prime}\right), 1.75$ (2H, quintet, $\left.J=7.3 \mathrm{~Hz}, \mathrm{H}-3^{\prime}\right), 1.40$ $\left(4 \mathrm{H}, \mathrm{m}, \mathrm{H}-4^{\prime}\right.$ and $\left.\mathrm{H}-5^{\prime}\right), 0.94\left(3 \mathrm{H}, \mathrm{t}, J=7.3 \mathrm{~Hz}, \mathrm{H}-6^{\prime}\right), 4.07\left(3 \mathrm{H}, \mathrm{s}, \mathrm{OCH}_{3}\right), 11.25(1 \mathrm{H}$, br.s, indole N$\mathrm{H}) ;{ }^{13} \mathrm{C}-\mathrm{NMR}\left(\mathrm{CDCl}_{3}\right): \delta 134.3$ (C-2), 138.0 (C-3), 130.6 (C-5), 113.8 (C-6), 116.0 (C-7), 131.7 (C-8), 129.3 (C-9), 107.6 (C-10), 163.8 (C-11), 109.9 (C-12), 145.0 (C-13), 16.1 (C-14), 203.0 (C-1'), 44.2 (C-2'), 23.2 (C-3'), 31.1 (C-4'), 22.9 (C-5'), $14.2\left(\mathrm{C}-6^{\prime}\right), 56.8\left(\mathrm{OCH}_{3}\right) ;$ HREIMS (m/z): 310.1690 $\left[\mathrm{C}_{19} \mathrm{H}_{22} \mathrm{~N}_{2} \mathrm{O}_{2}\right.$ calcd. 310.1681]; EIMS m/z (\%): 310 [M $\left.\mathrm{M}^{+}\right]$(26), 267 (6), 254 (11), 239 (100), 224 (12), $211(6), 196(8)$.

12-Heptanoyl-11-methoxy-3-methyl- $\beta$-carboline (16). $18.0 \mathrm{mg} ; \mathrm{mp}: 296-297{ }^{\circ} \mathrm{C}$; IR $\left(\mathrm{CHCl}_{3}\right) v_{\max } \mathrm{cm}^{-1}$ : 3384.5 (indole N-H), 2926.1, $2857.4(\mathrm{C}-\mathrm{H}), 1648.2$ (ketone $\mathrm{C}=\mathrm{O}$ ), 1608.2, 1542.9, 1455.2 (aromatic $\mathrm{C}=\mathrm{C}), 1151.2(\mathrm{C}-\mathrm{O})$; UV $(\mathrm{MeOH}) \lambda_{\max } \mathrm{nm}: 361.8,321.2,269.3,232.1 ;{ }^{1} \mathrm{H}-\mathrm{NMR}\left(\mathrm{CDCl}_{3}\right): \delta 8.35(1 \mathrm{H}$, d, $J=4.7 \mathrm{~Hz}, \mathrm{H}-5), 7.85(1 \mathrm{H}, \mathrm{d}, J=4.7 \mathrm{~Hz}, \mathrm{H}-6), 8.27(1 \mathrm{H}, \mathrm{d}, J=8.7 \mathrm{~Hz}, \mathrm{H}-9), 7.02$ (1H, d, $J=8.7$ Hz, H-10), 3.05 (3H, s, H-14), 3.15 (2H, t, $\left.J=7.3 \mathrm{~Hz}, \mathrm{H}-2^{\prime}\right), 1.74$ (2H, quintet, $\left.J=7.3 \mathrm{~Hz}, \mathrm{H}-3^{\prime}\right), 1.40$ ( $2 \mathrm{H}$, quintet, $\left.J=7.3 \mathrm{~Hz}, \mathrm{H}-4^{\prime}\right), 1.34\left(4 \mathrm{H}, \mathrm{m}, \mathrm{H}-5^{\prime}\right.$ and $\left.\mathrm{H}-6^{\prime}\right), 0.89\left(3 \mathrm{H}, \mathrm{t}, J=7.3 \mathrm{~Hz}, \mathrm{H}-7^{\prime}\right), 4.11(3 \mathrm{H}$, $\left.\mathrm{s}, \mathrm{OCH}_{3}\right), 11.17(1 \mathrm{H}$, br.s, indole $\mathrm{N}-\mathrm{H}) ;{ }^{13} \mathrm{C}-\mathrm{NMR}\left(\mathrm{CDCl}_{3}\right)$ : $\delta 134.2(\mathrm{C}-2), 137.9(\mathrm{C}-3), 131.0(\mathrm{C}-5)$, 114.0 (C-6), 115.7 (C-7), 131.8 (C-8), 129.4 (C-9), 107.5 (C-10), 163.8 (C-11), 110.0 (C-12), 145.1 (C-13), 16.0 (C-14), 203.4 (C-1'), 44.3 (C-2'), 24.1 (C-3'), 30.2 (C-4'), 32.2 (C-5'), 23.2 (C-6'), 14.4 $\left(\mathrm{C}-7^{\prime}\right), 56.9\left(\mathrm{OCH}_{3}\right)$; HREIMS $(\mathrm{m} / \mathrm{z}): 324.1826\left[\mathrm{C}_{20} \mathrm{H}_{24} \mathrm{~N}_{2} \mathrm{O}_{2}\right.$ calcd. 324.1838$]$; EIMS $m / z(\%): 324$ $\left[\mathrm{M}^{+}\right](84), 267$ (53), 254 (66), 239 (100), 224 (12), 211 (7), 196 (8).

12-Capryloyl-11-methoxy-3-methyl- $\beta$-carboline (17). $18.3 \mathrm{mg}$; mp: $296-297{ }^{\circ} \mathrm{C}$; IR $\left(\mathrm{CHCl}_{3}\right) v_{\max } \mathrm{cm}^{-1}$ : 3382.9 (indole $\mathrm{N}-\mathrm{H}$ ), 2923.9, $2854.5(\mathrm{C}-\mathrm{H}), 1647.1$ (ketone $\mathrm{C}=\mathrm{O}$ ), 1612.1, 1545.6, 1458.1 (aromatic $\mathrm{C}=\mathrm{C}), 1153.2(\mathrm{C}-\mathrm{O})$; UV $(\mathrm{MeOH}) \lambda_{\max } \mathrm{nm}: 361.2,321.5,269.3,232.4 ;{ }^{1} \mathrm{H}-\mathrm{NMR}\left(\mathrm{CDCl}_{3}\right): \delta 8.35(1 \mathrm{H}$, $\mathrm{d}, J=5.2 \mathrm{~Hz}, \mathrm{H}-5), 7.83(1 \mathrm{H}, \mathrm{d}, J=5.2 \mathrm{~Hz}, \mathrm{H}-6), 8.25(1 \mathrm{H}, \mathrm{d}, J=8.7 \mathrm{~Hz}, \mathrm{H}-9), 7.01(1 \mathrm{H}, \mathrm{d}, J=8.7$ Hz, H-10), 3.02 (3H, s, H-14), 3.16 (2H, t, $\left.J=7.3 \mathrm{~Hz}, \mathrm{H}-2^{\prime}\right), 1.74$ (2H, quintet, $\left.J=7.3 \mathrm{~Hz}, \mathrm{H}-3^{\prime}\right), 1.37$ (2H, quintet, $\left.J=7.3 \mathrm{~Hz}, \mathrm{H}-4^{\prime}\right), 1.30\left(6 \mathrm{H}, \mathrm{m}, \mathrm{H}-5^{\prime}, \mathrm{H}-6^{\prime}\right.$ and $\left.\mathrm{H}-7^{\prime}\right), 0.86\left(3 \mathrm{H}, \mathrm{t}, J=7.1 \mathrm{~Hz}, \mathrm{H}-8^{\prime}\right), 4.12$ $\left(3 \mathrm{H}, \mathrm{s}, \mathrm{OCH}_{3}\right), 11.23(1 \mathrm{H}$, br.s, indole $\mathrm{N}-\mathrm{H}) ;{ }^{13} \mathrm{C}-\mathrm{NMR}\left(\mathrm{CDCl}_{3}\right): \delta 134.2(\mathrm{C}-2), 137.7(\mathrm{C}-3), 130.4(\mathrm{C}-$ 5), 114.0 (C-6), 115.7 (C-7), 132.3 (C-8), 129.5 (C-9), 107.8 (C-10), 163.9 (C-11), 110.2 (C-12), 145.1 (C-13), 15.7 (C-14), $203.1\left(\mathrm{C}-1^{\prime}\right), 45.4\left(\mathrm{C}-2^{\prime}\right), 24.3\left(\mathrm{C}-3^{\prime}\right), 29.4\left(\mathrm{C}-4^{\prime}\right)^{*}, 29.3\left(\mathrm{C}-5^{\prime}\right) *, 31.8$ (C-6'), 22.6 $\left(\mathrm{C}-7^{\prime}\right), 14.1\left(\mathrm{C}-8^{\prime}\right), 56.9\left(\mathrm{OCH}_{3}\right)$ (* values may be interchanged); HREIMS $(\mathrm{m} / \mathrm{z})$ : 338.1988 $\left[\mathrm{C}_{21} \mathrm{H}_{26} \mathrm{~N}_{2} \mathrm{O}_{2}\right.$ calcd. 338.1994]; EIMS m/z (\%): 338 [M $]$ (70), 267 (18), 254 (48), 239 (100), 224 (10), $211(5), 196(5)$. 
10-Propionyl-11-hydroxy-3-methyl- $\beta$-carboline (18). $28.5 \mathrm{mg} ; \mathrm{mp}$ : $322-323{ }^{\circ} \mathrm{C}$; IR $\left(\mathrm{CHCl}_{3}\right) v_{\max } \mathrm{cm}^{-1}$ : $3378.2(\mathrm{OH}$ and indole $\mathrm{N}-\mathrm{H}), 2932.3,2845.2(\mathrm{C}-\mathrm{H}), 1646.8$ (ketone $\mathrm{C}=\mathrm{O}), 1612.1,1535.3,1443.1$ (aromatic $\mathrm{C}=\mathrm{C}), 1153.4(\mathrm{C}-\mathrm{O})$; UV $(\mathrm{MeOH}) \lambda_{\max } \mathrm{nm}$ : 347.2, 340.4, 275.2, 236.3; ${ }^{1} \mathrm{H}-\mathrm{NMR}\left(\mathrm{CD}_{3} \mathrm{OD}\right)$ : Table 2; ${ }^{13} \mathrm{C}-\mathrm{NMR}\left(\mathrm{CD}_{3} \mathrm{OD}\right)$ : Table 3; HREIMS $(\mathrm{m} / \mathrm{z})$ : $254.1070\left[\mathrm{C}_{15} \mathrm{H}_{14} \mathrm{~N}_{2} \mathrm{O}_{2}\right.$ calcd. 254.1055]; EIMS $m / z(\%): 254\left[\mathrm{M}^{+}\right](79), 236(9), 225$ (100), 197 (6).

10-Butyryl-11-hydroxy-3-methyl- $\beta$-carboline (19). $8.8 \mathrm{mg}$; mp: $323-324{ }^{\circ} \mathrm{C}$; IR $\left(\mathrm{CHCl}_{3}\right) v_{\max } \mathrm{cm}^{-1}$ : $3379.5(\mathrm{OH}$ and indole $\mathrm{N}-\mathrm{H}), 2954.3,2863.1(\mathrm{C}-\mathrm{H}), 1646.6$ (ketone $\mathrm{C}=\mathrm{O}), 1608.7,1536.5,1432.1$ (aromatic $\mathrm{C}=\mathrm{C}), 1158.3(\mathrm{C}-\mathrm{O})$; UV $(\mathrm{MeOH}) \lambda_{\max } \mathrm{nm}: 347.4,340.2,275.3,236.5 ;{ }^{1} \mathrm{H}-\mathrm{NMR}\left(\mathrm{CD}_{3} \mathrm{OD}\right)$ : $\delta 8.22(1 \mathrm{H}, \mathrm{d}, J=5.6 \mathrm{~Hz}, \mathrm{H}-5), 8.08(1 \mathrm{H}, \mathrm{d}, J=5.6 \mathrm{~Hz}, \mathrm{H}-6), 8.89(1 \mathrm{H}, \mathrm{s}, \mathrm{H}-9), 6.94(1 \mathrm{H}, \mathrm{s}, \mathrm{H}-12)$, $2.82(3 \mathrm{H}, \mathrm{s}, \mathrm{H}-14), 3.20$ (2H, t, $\left.J=7.3 \mathrm{~Hz}, \mathrm{H}-2^{\prime}\right), 1.85$ (2H, sextet, $\left.J=7.4 \mathrm{~Hz}, \mathrm{H}-3^{\prime}\right), 1.08$ (3H, t, $J=$ $\left.7.3 \mathrm{~Hz}, \mathrm{H}-4{ }^{\prime}\right) ;{ }^{13} \mathrm{C}-\mathrm{NMR}\left(\mathrm{CD}_{3} \mathrm{OD}\right): \delta 137.2$ (C-2), 142.3 (C-3), 139.2 (C-5), 113.6 (C-6), 115.7 (C-7), 131.3 (C-8), 127.2 (C-9), 116.2 (C-10), 164.2 (C-11), 98.5 (C-12), 147.9 (C-13), 19.3 (C-14), 208.0 $\left(\mathrm{C}-1^{\prime}\right), 38.2$ (C-2'), 18.7 (C-3'), 14.3 (C-4'); HREIMS (m/z): 268.1220 [ $\mathrm{C}_{16} \mathrm{H}_{16} \mathrm{~N}_{2} \mathrm{O}_{2}$ calcd. 268.1212]; EIMS $m / z(\%): 268\left[\mathrm{M}^{+}\right](28), 253$ (7), $240(20), 225$ (100), 197 (4).

10-Isobutyryl-11-hydroxy-3-methyl- $\beta$-carboline (20). $20.2 \mathrm{mg}$; mp: 326-327 ${ }^{\circ} \mathrm{C}$; IR $\left(\mathrm{CHCl}_{3}\right) v_{\max } \mathrm{cm}^{-1}$ : $3378.2(\mathrm{OH}$ and indole $\mathrm{N}-\mathrm{H}), 2925.1,2854.9(\mathrm{C}-\mathrm{H}), 1647.2$ (ketone $\mathrm{C}=\mathrm{O}), 1608.1,1537.1,1458.3$ (aromatic $\mathrm{C}=\mathrm{C}), 1158.2(\mathrm{C}-\mathrm{O})$; UV $(\mathrm{MeOH}) \lambda_{\max } \mathrm{nm}$ : 347.5, 340.2, 275.1, 236.4; ${ }^{1} \mathrm{H}-\mathrm{NMR}\left(\mathrm{CD}_{3} \mathrm{OD}\right)$ : $\delta 8.18(1 \mathrm{H}, \mathrm{d}, J=5.4 \mathrm{~Hz}, \mathrm{H}-5), 7.97(1 \mathrm{H}, \mathrm{d}, J=5.4 \mathrm{~Hz}, \mathrm{H}-6), 8.83(1 \mathrm{H}, \mathrm{s}, \mathrm{H}-9), 6.91(1 \mathrm{H}, \mathrm{s}, \mathrm{H}-12)$, $2.77(3 \mathrm{H}, \mathrm{s}, \mathrm{H}-14), 3.93\left(1 \mathrm{H}\right.$, septet, $\left.J=6.8 \mathrm{~Hz}, \mathrm{H}-2^{\prime}\right), 1.29\left(6 \mathrm{H}, \mathrm{d}, J=6.8 \mathrm{~Hz}, \mathrm{H}-3{ }^{\prime}\right.$ and $\left.\mathrm{H}-4^{\prime}\right) ;{ }^{13} \mathrm{C}-$ NMR (CD $\left.{ }_{3} \mathrm{OD}\right): \delta 137.4$ (C-2), 141.8 (C-3), 138.7 (C-5), 114.2 (C-6), 115.0 (C-7), 131.6 (C-8), 127.4 (C-9), 116.2 (C-10), 165.4 (C-11), 98.9 (C-12), 148.3 (C-13), 18.8 (C-14), 212.0 (C-1'), 35.9 (C-2'), 20.1 (C-3' and C-4'); HREIMS (m/z): $268.1218\left[\mathrm{C}_{16} \mathrm{H}_{16} \mathrm{~N}_{2} \mathrm{O}_{2}\right.$ calcd. 268.1212]; EIMS $m / z(\%): 268$ $\left[\mathrm{M}^{+}\right]$(97), 253 (12), 225 (100), 197 (4).

10-Hexanoyl-11-hydroxy-3-methyl- $\beta$-carboline (21). $14.2 \mathrm{mg}$; mp: 327-328 ${ }^{\circ} \mathrm{C}$; IR $\left(\mathrm{CHCl}_{3}\right) v_{\max } \mathrm{cm}^{-1}$ : $3373.2(\mathrm{OH}$ and indole $\mathrm{N}-\mathrm{H}), 2923.9,2854.5(\mathrm{C}-\mathrm{H}), 1645.2$ (ketone $\mathrm{C}=\mathrm{O}), 1610.8,1536.6,1461.9$ (aromatic $\mathrm{C}=\mathrm{C}), 1199.6(\mathrm{C}-\mathrm{O})$; UV $(\mathrm{MeOH}) \lambda_{\max } \mathrm{nm}: 347.6,340.0,275.0,236.2 ;{ }^{1} \mathrm{H}-\mathrm{NMR}\left(\mathrm{CD}_{3} \mathrm{OD}\right)$ : $\delta 8.20(1 \mathrm{H}, \mathrm{d}, J=5.2 \mathrm{~Hz}, \mathrm{H}-5), 8.00(1 \mathrm{H}, \mathrm{d}, J=5.2 \mathrm{~Hz}, \mathrm{H}-6), 8.83(1 \mathrm{H}, \mathrm{s}, \mathrm{H}-9), 6.91(1 \mathrm{H}, \mathrm{s}, \mathrm{H}-12)$, $2.78(3 \mathrm{H}, \mathrm{s}, \mathrm{H}-14), 3.20\left(2 \mathrm{H}, \mathrm{t}, J=7.3 \mathrm{~Hz}, \mathrm{H}-2^{\prime}\right), 1.80\left(2 \mathrm{H}\right.$, quintet, $\left.J=7.3 \mathrm{~Hz}, \mathrm{H}-3^{\prime}\right), 1.43$ (4H, m, H$4^{\prime}$ and $\left.\mathrm{H}-5^{\prime}\right), 0.95\left(3 \mathrm{H}, \mathrm{t}, J=7.3 \mathrm{~Hz}, \mathrm{H}-6{ }^{\prime}\right) ;{ }^{13} \mathrm{C}-\mathrm{NMR}\left(\mathrm{CD}_{3} \mathrm{OD}\right): \delta 137.4(\mathrm{C}-2), 142.5(\mathrm{C}-3), 139.5(\mathrm{C}-$ 5), 113.8 (C-6), 115.7 (C-7), 131.2 (C-8), 127.3 (C-9), 116.4 (C-10), 164.6 (C-11), 98.5 (C-12), 147.9 (C-13), 19.5 (C-14), 208.1 (C-1'), 38.9 (C-2'), 25.8 (C-3'), $32.6\left(\mathrm{C}-4^{\prime}\right), 23.6\left(\mathrm{C}-5^{\prime}\right), 14.3\left(\mathrm{C}-6^{\prime}\right)$; HREIMS (m/z): 296.1518 [ $\mathrm{C}_{18} \mathrm{H}_{20} \mathrm{~N}_{2} \mathrm{O}_{2}$ calcd. 296.1525]; EIMS m/z (\%): $296\left[\mathrm{M}^{+}\right]$(34), 278 (10), 253 (17), 240 (25), 225 (100), 197 (6).

10-Heptanoyl-11-hydroxy-3-methyl- $\beta$-carboline (22). $8.6 \mathrm{mg} ; \mathrm{mp}: 329-330{ }^{\circ} \mathrm{C}$; IR $\left(\mathrm{CHCl}_{3}\right) v_{\max } \mathrm{cm}^{-1}$ : $3375.4(\mathrm{OH}$ and indole N-H), 2924.2, $2856.1(\mathrm{C}-\mathrm{H}), 1646.4$ (ketone $\mathrm{C}=\mathrm{O}), 1608.7,1536.2,1422.7$ (aromatic $\mathrm{C}=\mathrm{C}), 1182.1(\mathrm{C}-\mathrm{O})$; UV $(\mathrm{MeOH}) \lambda_{\max } \mathrm{nm}: 347.1,339.9,275.1,236.3 ;{ }^{1} \mathrm{H}-\mathrm{NMR}\left(\mathrm{CD}_{3} \mathrm{OD}\right)$ : $\delta 8.17(1 \mathrm{H}, \mathrm{d}, J=5.7 \mathrm{~Hz}, \mathrm{H}-5), 7.97(1 \mathrm{H}, \mathrm{d}, J=5.7 \mathrm{~Hz}, \mathrm{H}-6), 8.88$ (1H, s, H-9), 6.92 (1H, s, H-12), $2.85(3 \mathrm{H}, \mathrm{s}, \mathrm{H}-14), 3.07\left(2 \mathrm{H}, \mathrm{t}, J=7.3 \mathrm{~Hz}, \mathrm{H}-2^{\prime}\right), 1.72\left(2 \mathrm{H}\right.$, quintet, $\left.J=7.3 \mathrm{~Hz}, \mathrm{H}-3^{\prime}\right), 1.37(2 \mathrm{H}$, 
quintet, $\left.J=7.3 \mathrm{~Hz}, \mathrm{H}-4^{\prime}\right), 1.27\left(4 \mathrm{H}, \mathrm{m}, \mathrm{H}-5^{\prime}\right.$ and $\left.\mathrm{H}-6^{\prime}\right), 0.81\left(3 \mathrm{H}, \mathrm{t}, J=7.3 \mathrm{~Hz}, \mathrm{H}-7^{\prime}\right) ;{ }^{13} \mathrm{C}-\mathrm{NMR}$ (CD $\left.{ }_{3} \mathrm{OD}\right): \delta 137.5$ (C-2), 142.3 (C-3), 139.5 (C-5), 113.7 (C-6), 115.8 (C-7), 131.1 (C-8), 127.2 (C-9), 116.3 (C-10), 164.3 (C-11), 98.4 (C-12), 147.6 (C-13), 19.4 (C-14), 207.9 (C-1'), 38.4 (C-2'), 24.4 (C3'), 29.2 (C-4'), 31.9 (C-5'), 22.9 (C-6'), 14.7 (C-7'); HREIMS (m/z): $310.1673\left[\mathrm{C}_{19} \mathrm{H}_{22} \mathrm{~N}_{2} \mathrm{O}_{2}\right.$ calcd. 310.1681]; EIMS m/z (\%): $310\left[\mathrm{M}^{+}\right]$(79), 292 (55), 253 (57), 240 (35), 225 (100), 197 (10).

10,12-Diacetyl-11-hydroxy-3-methyl- $\beta$-carboline (23). $17.0 \mathrm{mg}$; $\mathrm{mp}: 328-329{ }^{\circ} \mathrm{C}$; IR $\left(\mathrm{CHCl}_{3}\right) v_{\max }$ $\mathrm{cm}^{-1}$ : $3384.2(\mathrm{OH}$ and indole $\mathrm{N}-\mathrm{H}), 2928.0,2850.1(\mathrm{C}-\mathrm{H}), 1644.2$ (ketone $\left.\mathrm{C}=\mathrm{O}\right), 1582.4,1494.6$, 1426.5 (aromatic $\mathrm{C}=\mathrm{C}), 1189.7(\mathrm{C}-\mathrm{O})$; $\mathrm{UV}(\mathrm{MeOH}) \lambda_{\max } \mathrm{nm}: 364.5,279.7,230.2 ;{ }^{1} \mathrm{H}-\mathrm{NMR}\left(\mathrm{CDCl}_{3}\right)$ : $\delta 8.40(1 \mathrm{H}, \mathrm{d}, J=5.3 \mathrm{~Hz}, \mathrm{H}-5), 7.79(1 \mathrm{H}, \mathrm{d}, J=5.3 \mathrm{~Hz}, \mathrm{H}-6), 8.69$ (1H, s, H-9), 2.88 (3H, s, H-14), $2.78\left(3 \mathrm{H}, \mathrm{s}, \mathrm{H}-2^{\prime}\right), 2.84\left(3 \mathrm{H}, \mathrm{s}, \mathrm{H}-2^{\prime \prime}\right), 14.67(1 \mathrm{H}, \mathrm{s}, \mathrm{OH}), 11.33(1 \mathrm{H}$, br.s, indole $\mathrm{N}-\mathrm{H}) ;{ }^{13} \mathrm{C}-\mathrm{NMR}$ $\left(\mathrm{CDCl}_{3}\right): \delta 135.1$ (C-2), 140.9 (C-3), 137.9 (C-5), 112.9 (C-6), 114.5 (C-7), 130.2 (C-8), 130.5 (C-9), 114.6 (C-10), 166.9 (C-11), 109.0 (C-12), 146.6 (C-13), 18.5 (C-14), 206.6 (C-1'), 32.2 (C-2'), 203.3 $\left(\mathrm{C}-1^{\prime \prime}\right), 36.9\left(\mathrm{C}-2^{\prime \prime}\right)$; HREIMS (m/z): 282.1012 [ $\mathrm{C}_{16} \mathrm{H}_{14} \mathrm{~N}_{2} \mathrm{O}_{3}$ calcd. 282.1004]; EIMS m/z (\%): 282 $\left[\mathrm{M}^{+}\right]$(91), 267 (100), 251 (18), 249 (32), 239 (4), 237 (18).

10,12-Dipropionyl-11-hydroxy-3-methyl- $\beta$-carboline (24). $16.6 \mathrm{mg}$; mp: 330-331 ${ }^{\circ} \mathrm{C}$; IR $\left(\mathrm{CHCl}_{3}\right) v_{\max }$ $\mathrm{cm}^{-1}: 3383.2(\mathrm{OH}$ and indole $\mathrm{N}-\mathrm{H}), 2927.2,2851.0(\mathrm{C}-\mathrm{H}), 1646.1$ (ketone $\left.\mathrm{C}=\mathrm{O}\right), 1580.4,1493.8$, 1461.3 (aromatic $\mathrm{C}=\mathrm{C}$ ), $1189.2(\mathrm{C}-\mathrm{O})$; UV (MeOH) $\lambda_{\max } \mathrm{nm}: 366.2,279.8,230.2 ;{ }^{1} \mathrm{H}-\mathrm{NMR}\left(\mathrm{CD}_{3} \mathrm{OD}\right)$ : Table 2; ${ }^{13} \mathrm{C}-\mathrm{NMR}\left(\mathrm{CD}_{3} \mathrm{OD}\right)$ : Table 3; HREIMS $(\mathrm{m} / \mathrm{z}): 310.1325\left[\mathrm{C}_{18} \mathrm{H}_{18} \mathrm{~N}_{2} \mathrm{O}_{3}\right.$ calcd. 310.1317$]$; EIMS $m / z(\%): 310\left[\mathrm{M}^{+}\right]$(100), 292 (12), 281 (98), 253 (5), 251 (19), 237 (24).

10,12-Dibutyryl-11-hydroxy-3-methyl- $\beta$-carboline (25). $15.5 \mathrm{mg} ; \mathrm{mp}: 331-332{ }^{\circ} \mathrm{C}$; IR $\left(\mathrm{CHCl}_{3}\right) v_{\max }$ $\mathrm{cm}^{-1}: 3383.5(\mathrm{OH}$ and indole $\mathrm{N}-\mathrm{H}), 2926.5,2856.5(\mathrm{C}-\mathrm{H}), 1646.2$ (ketone $\left.\mathrm{C}=\mathrm{O}\right), 1580.2,1494.3$, 1416.2 (aromatic $\mathrm{C}=\mathrm{C}), 1188.5(\mathrm{C}-\mathrm{O})$; $\mathrm{UV}(\mathrm{MeOH}) \lambda_{\max } \mathrm{nm}: 366.2,279.7,230.0 ;{ }^{1} \mathrm{H}-\mathrm{NMR}\left(\mathrm{CDCl}_{3}\right)$ : $\delta 8.41(1 \mathrm{H}, \mathrm{d}, J=5.3 \mathrm{~Hz}, \mathrm{H}-5), 7.82(1 \mathrm{H}, \mathrm{d}, J=5.3 \mathrm{~Hz}, \mathrm{H}-6), 8.69$ (1H, s, H-9), 2.87 (3H, s, H-14), $3.14\left(2 \mathrm{H}, \mathrm{t}, J=7.3 \mathrm{~Hz}, \mathrm{H}-2^{\prime}\right), 1.88$ (2H, sextet, $\left.J=7.3 \mathrm{~Hz}, \mathrm{H}-3^{\prime}\right), 1.08$ (3H, t, H-4'), 3.24 (2H, t, $J=$ $\left.7.3 \mathrm{~Hz}, \mathrm{H}-2^{\prime \prime}\right), 1.79$ (2H, sextet, $\left.J=7.3 \mathrm{~Hz}, \mathrm{H}-3^{\prime \prime}\right), 1.04$ (3H, t, $\left.J=7.3 \mathrm{~Hz}, \mathrm{H}-4^{\prime \prime}\right), 14.74$ (1H, s, OH), $11.28(1 \mathrm{H}$, br.s, indole $\mathrm{N}-\mathrm{H}) ;{ }^{13} \mathrm{C}-\mathrm{NMR}\left(\mathrm{CDCl}_{3}\right): \delta 135.2(\mathrm{C}-2), 141.2(\mathrm{C}-3), 137.7(\mathrm{C}-5), 112.8(\mathrm{C}-6)$, $114.6(\mathrm{C}-7)^{*}, 130.6$ (C-8 and C-9), 114.8 (C-10)*, 167.0 (C-11), 108.9 (C-12), 146.7 (C-13), 18.5 (C14), $206.7\left(\mathrm{C}-1^{\prime}\right), 40.3$ (C-2'), 18.3 (C-3'), 14.0 (C-4')**, 203.1 (C-1"'), 47.2 (C-2"'), 17.6 (C-3"'), 13.9 $\left(\mathrm{C}-4{ }^{\prime \prime}\right) * *$ (*** values may be interchanged); HREIMS (m/z): $338.1625\left[\mathrm{C}_{20} \mathrm{H}_{22} \mathrm{~N}_{2} \mathrm{O}_{3}\right.$ calcd. 338.1630]; EIMS m/z (\%): 338 [M ] (63), 323 (10), 295 (100), 282 (30), 277 (24), 267 (16), 251 (16), 237 (22).

10,12-Diisobutyryl-11-hydroxy-3-methyl- $\beta$-carboline (26). $12.6 \mathrm{mg}$; mp: 333-334 ${ }^{\circ} \mathrm{C}$; IR $\left(\mathrm{CHCl}_{3}\right) v_{\max }$ $\mathrm{cm}^{-1}: 3385.1(\mathrm{OH}$ and indole $\mathrm{N}-\mathrm{H}), 2926.0,2854.0(\mathrm{C}-\mathrm{H}), 1645.1$ (ketone $\left.\mathrm{C}=\mathrm{O}\right), 1581.2,1494.5$, 1455.2 (aromatic $\mathrm{C}=\mathrm{C}), 1187.2(\mathrm{C}-\mathrm{O})$; $\mathrm{UV}(\mathrm{MeOH}) \lambda_{\max } \mathrm{nm}: 365.8,279.8,230.1 ;{ }^{1} \mathrm{H}-\mathrm{NMR}\left(\mathrm{CDCl}_{3}\right)$ : $\delta 8.41(1 \mathrm{H}, \mathrm{d}, J=5.3 \mathrm{~Hz}, \mathrm{H}-5), 7.82(1 \mathrm{H}, \mathrm{d}, J=5.3 \mathrm{~Hz}, \mathrm{H}-6), 8.75(1 \mathrm{H}, \mathrm{s}, \mathrm{H}-9), 2.88$ (3H, s, H-14), $3.89\left(1 \mathrm{H}\right.$, septet, $\left.J=7.3 \mathrm{~Hz}, \mathrm{H}-2^{\prime}\right), 1.33\left(6 \mathrm{H}, \mathrm{d}, J=7.3 \mathrm{~Hz}, \mathrm{H}-3^{\prime}\right.$ and $\left.\mathrm{H}-4^{\prime}\right), 3.98(1 \mathrm{H}$, septet, $J=7.3$ $\left.\mathrm{Hz}, \mathrm{H}-2^{\prime \prime}\right), 1.26\left(6 \mathrm{H}, \mathrm{d}, J=7.3 \mathrm{~Hz}, \mathrm{H}-3^{\prime \prime}\right.$ and H-4"), $14.71(1 \mathrm{H}, \mathrm{s}, \mathrm{OH}), 11.41$ (1H, br.s, indole N-H); ${ }^{13} \mathrm{C}-\mathrm{NMR}\left(\mathrm{CDCl}_{3}\right): \delta 135.1(\mathrm{C}-2), 141.1(\mathrm{C}-3), 137.8(\mathrm{C}-5), 112.9(\mathrm{C}-6), 114.4(\mathrm{C}-7), 130.2(\mathrm{C}-8)$, 130.7 (C-9), 114.5 (C-10), 166.9 (C-11), 108.8 (C-12), 146.5 (C-13), 18.6 (C-14), 211.8 (C-1'), 36.8 
(C-2'), 19.3 (C-3' and C-4'), 207.2 (C-1"), 40.4 (C-2"), 19.1 (C-3" and C-4"); HREIMS (m/z): $338.1644\left[\mathrm{C}_{20} \mathrm{H}_{22} \mathrm{~N}_{2} \mathrm{O}_{3}\right.$ calcd. 338.1630]; EIMS m/z (\%): 338 [M $]$ (36), 295 (100), 277 (45), 251 (22), 237 (26).

10,12-Divaleryl-11-hydroxy-3-methyl- $\beta$-carboline (27). $25.5 \mathrm{mg}$; $\mathrm{mp}: 332-333{ }^{\circ} \mathrm{C}$; IR $\left(\mathrm{CHCl}_{3}\right) v_{\max }$ $\mathrm{cm}^{-1}$ : $3383.0(\mathrm{OH}$ and indole $\mathrm{N}-\mathrm{H}), 2927.4,2862.8(\mathrm{C}-\mathrm{H}), 1644.2$ (ketone $\left.\mathrm{C}=\mathrm{O}\right), 1582.4,1495.3$, 1418.7 (aromatic $\mathrm{C}=\mathrm{C}), 1199.8(\mathrm{C}-\mathrm{O})$; $\mathrm{UV}(\mathrm{MeOH}) \lambda_{\max } \mathrm{nm}: 364.8,279.8,230.0 ;{ }^{1} \mathrm{H}-\mathrm{NMR}\left(\mathrm{CDCl}_{3}\right)$ : $\delta 8.43(1 \mathrm{H}, \mathrm{d}, J=5.3 \mathrm{~Hz}, \mathrm{H}-5), 7.89(1 \mathrm{H}, \mathrm{d}, J=5.3 \mathrm{~Hz}, \mathrm{H}-6), 8.74$ (1H, s, H-9), 2.93 (3H, s, H-14), $3.16\left(2 \mathrm{H}, \mathrm{t}, J=7.3 \mathrm{~Hz}, \mathrm{H}-2^{\prime}\right), 1.80$ (2H, quintet, $\left.J=7.3 \mathrm{~Hz}, \mathrm{H}-3^{\prime}\right), 1.49$ (2H, sextet, $\left.J=7.3 \mathrm{~Hz}, \mathrm{H}-4^{\prime}\right)$, $0.99\left(3 \mathrm{H}, \mathrm{t}, J=7.3 \mathrm{~Hz}, \mathrm{H}-5^{\prime}\right), 3.25\left(2 \mathrm{H}, \mathrm{t}, J=7.3 \mathrm{~Hz}, \mathrm{H}-2^{\prime \prime}\right), 1.74$ (2H, quintet, $\left.J=7.3 \mathrm{~Hz}, \mathrm{H}-3^{\prime \prime}\right)$, 1.45 (2H, sextet, $\left.J=7.3 \mathrm{~Hz}, \mathrm{H}-4^{\prime \prime}\right), 0.96\left(3 \mathrm{H}, \mathrm{t}, J=7.3 \mathrm{~Hz}, \mathrm{H}-5^{\prime \prime}\right), 14.75$ (1H, s, OH), 11.40 (1H, br.s, indole N-H); ${ }^{13} \mathrm{C}-\mathrm{NMR}\left(\mathrm{CDCl}_{3}\right): \delta 135.2(\mathrm{C}-2), 141.0$ (C-3), 137.7 (C-5), 112.8 (C-6), $114.6(\mathrm{C}-7)$, 130.0 (C-8), 130.6 (C-9), 114.6 (C-10), 166.9 (C-11), 109.0 (C-12), 146.6 (C-13), 18.5 (C-14), 206.8 $\left(\mathrm{C}-1^{\prime}\right), 38.1\left(\mathrm{C}-2^{\prime}\right), 26.3\left(\mathrm{C}-3^{\prime}\right), 22.5\left(\mathrm{C}-4^{\prime}\right)^{*}, 14.0\left(\mathrm{C}-4^{\prime}\right)^{* *}, 203.3\left(\mathrm{C}-1^{\prime \prime}\right), 44.9\left(\mathrm{C}-2^{\prime \prime}\right), 26.9(\mathrm{C}-$ $\left.3^{\prime \prime}\right), 22.4\left(\mathrm{C}-4^{\prime \prime}\right)^{*}, 13.9\left(\mathrm{C}-5^{\prime \prime}\right)^{* *}$ (*** values may be inter-changed); HREIMS $(\mathrm{m} / \mathrm{z})$ : 366.1935 [C $\mathrm{C}_{22} \mathrm{H}_{26} \mathrm{~N}_{2} \mathrm{O}_{3}$ calcd. 366.1943]; EIMS m/z (\%): 366 [M $\mathrm{M}^{+}$(20), 348 (24), 337 (77), 324 (25), 309 (100), 295 (22), 282 (16), 267 (20), 251 (18), 237 (25).

10,12-Dihexanoyl-11-hydroxy-3-methyl- $\beta$-carboline (28). $25.1 \mathrm{mg}$; mp: $335-336{ }^{\circ} \mathrm{C}$; IR $\left(\mathrm{CHCl}_{3}\right) v_{\max }$ $\mathrm{cm}^{-1}$ : $3384.2(\mathrm{OH}$ and indole $\mathrm{N}-\mathrm{H}), 2924.6,2855.2(\mathrm{C}-\mathrm{H}), 1645.2$ (ketone $\left.\mathrm{C}=\mathrm{O}\right), 1581.2,1494.8$, 1456.3 (aromatic $\mathrm{C}=\mathrm{C}), 1189.2(\mathrm{C}-\mathrm{O})$; $\mathrm{UV}(\mathrm{MeOH}) \lambda_{\max } \mathrm{nm}: 364.9,279.6,230.2 ;{ }^{1} \mathrm{H}-\mathrm{NMR}\left(\mathrm{CDCl}_{3}\right)$ : $\delta 8.40(1 \mathrm{H}, \mathrm{d}, J=5.2 \mathrm{~Hz}, \mathrm{H}-5), 7.81(1 \mathrm{H}, \mathrm{d}, J=5.2 \mathrm{~Hz}, \mathrm{H}-6), 8.70(1 \mathrm{H}, \mathrm{s}, \mathrm{H}-9), 2.89$ (3H, s, H-14), $3.10\left(2 \mathrm{H}, \mathrm{t}, J=7.3 \mathrm{~Hz}, \mathrm{H}-2^{\prime}\right), 1.81\left(2 \mathrm{H}\right.$, quintet, $\left.J=7.3 \mathrm{~Hz}, \mathrm{H}-3^{\prime}\right), 1.36\left(8 \mathrm{H}, \mathrm{m}, \mathrm{H}-4^{\prime}, \mathrm{H}-5^{\prime}, \mathrm{H}-4^{\prime \prime}\right.$ and H-5'), $0.93\left(3 \mathrm{H}, \mathrm{t}, J=6.8 \mathrm{~Hz}, \mathrm{H}-6^{\prime}\right), 3.21\left(2 \mathrm{H}, \mathrm{t}, J=7.3 \mathrm{~Hz}, \mathrm{H}-2^{\prime \prime}\right), 1.74(2 \mathrm{H}$, quintet, $J=7.3 \mathrm{~Hz}, \mathrm{H}-$ $\left.3^{\prime \prime}\right), 0.91\left(3 \mathrm{H}, \mathrm{t}, J=6.8 \mathrm{~Hz}, \mathrm{H}-6^{\prime \prime}\right), 14.69(1 \mathrm{H}, \mathrm{s}, \mathrm{OH}), 11.31(1 \mathrm{H}$, br.s, indole $\mathrm{N}-\mathrm{H}) ;{ }^{13} \mathrm{C}-\mathrm{NMR}$ $\left(\mathrm{CDCl}_{3}\right): \delta 135.0(\mathrm{C}-2), 141.1(\mathrm{C}-3), 137.9$ (C-5), 112.8 (C-6), 114.5 (C-7), 130.0 (C-8), 130.6 (C-9), 114.7 (C-10), 167.0 (C-11), 108.9 (C-12), 146.4 (C-13), 18.4 (C-14), 206.6 (C-1'), 38.3 (C-2'), 25.7 $\left(\mathrm{C}-3^{\prime}\right), 32.9\left(\mathrm{C}-4^{\prime}\right)^{*}, 23.8\left(\mathrm{C}-5^{\prime}\right)^{* *}, 14.2\left(\mathrm{C}-6^{\prime}\right)^{\psi}, 203.5\left(\mathrm{C}-1^{\prime \prime}\right), 45.1\left(\mathrm{C}-2^{\prime \prime}\right), 25.5\left(\mathrm{C}-3^{\prime \prime}\right), 32.8\left(\mathrm{C}-4^{\prime \prime}\right)^{*}$, $23.7\left(\mathrm{C}-5^{\prime \prime}\right)^{* *}, 14.1\left(\mathrm{C}-6^{\prime \prime}\right)^{\psi}\left(*{ }^{* *}{ }^{\Psi}\right.$ values may be inter-changed); HREIMS $(\mathrm{m} / \mathrm{z}): 394.2244$ [C ${ }_{24} \mathrm{H}_{30} \mathrm{~N}_{2} \mathrm{O}_{3}$ calcd. 394.2256]; EIMS m/z (\%): 394 [M $\left.{ }^{+}\right]$(100), 376 (32), 351 (87), 338 (6), 323 (98), 295 (30), 282 (19), 267 (13), 251 (21), 237 (18).

10,12-Diheptanoyl-11-hydroxy-3-methyl- $\beta$-carboline (29). $27.1 \mathrm{mg}$; mp: 336-337 ${ }^{\circ} \mathrm{C}$; IR $\left(\mathrm{CHCl}_{3}\right) v_{\max }$ $\mathrm{cm}^{-1}: 3383.8(\mathrm{OH}$ and indole $\mathrm{N}-\mathrm{H}), 2925.3,2855.4(\mathrm{C}-\mathrm{H}), 1644.2$ (ketone $\left.\mathrm{C}=\mathrm{O}\right), 1580.0,1495.5$, 1450.1 (aromatic $\mathrm{C}=\mathrm{C}), 1201.1(\mathrm{C}-\mathrm{O})$; $\mathrm{UV}(\mathrm{MeOH}) \lambda_{\max } \mathrm{nm}: 366.8,284.6,231.8 ;{ }^{1} \mathrm{H}-\mathrm{NMR}\left(\mathrm{CDCl}_{3}\right)$ : $\delta 8.43(1 \mathrm{H}, \mathrm{d}, J=5.3 \mathrm{~Hz}, \mathrm{H}-5), 7.85(1 \mathrm{H}, \mathrm{d}, J=5.3 \mathrm{~Hz}, \mathrm{H}-6), 8.72(1 \mathrm{H}, \mathrm{s}, \mathrm{H}-9), 2.92$ (3H, s, H-14), $3.15\left(2 \mathrm{H}, \mathrm{t}, J=7.3 \mathrm{~Hz}, \mathrm{H}-2^{\prime}\right), 1.82$ (2H, quintet, $\left.J=7.3 \mathrm{~Hz}, \mathrm{H}-3^{\prime}\right), 1.45$ (2H, m, H-4') 1.34 (8H, m, H5', H-6', H-5" and H-6"'), $0.90\left(3 \mathrm{H}, \mathrm{t}, J=6.8 \mathrm{~Hz}, \mathrm{H}-7^{\prime}\right), 3.25\left(2 \mathrm{H}, \mathrm{t}, J=7.3 \mathrm{~Hz}, \mathrm{H}-2^{\prime \prime}\right), 1.75(2 \mathrm{H}$, quintet, $\left.J=7.3 \mathrm{~Hz}, \mathrm{H}-3^{\prime \prime}\right), 1.42\left(2 \mathrm{H}, \mathrm{m}, \mathrm{H}-4^{\prime \prime}\right), 0.89$ (3H, t, $\left.J=6.8 \mathrm{~Hz}, \mathrm{H}-7^{\prime \prime}\right), 14.75(1 \mathrm{H}, \mathrm{s}, \mathrm{OH})$, $11.38(1 \mathrm{H}$, br.s, indole $\mathrm{N}-\mathrm{H}) ;{ }^{13} \mathrm{C}-\mathrm{NMR}\left(\mathrm{CDCl}_{3}\right): \delta 135.1(\mathrm{C}-2), 140.9(\mathrm{C}-3), 137.9(\mathrm{C}-5), 112.7(\mathrm{C}-6)$, 114.5 (C-7)*, 129.9 (C-8), 130.5 (C-9), 114.6 (C-10)*, 166.9 (C-11), 108.7 (C-12), 146.5 (C-13), 18.4

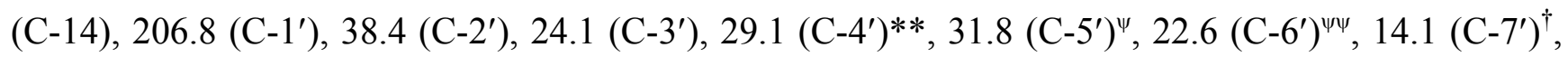


$203.3\left(\mathrm{C}-1^{\prime \prime}\right), 45.3\left(\mathrm{C}-2^{\prime \prime}\right), 24.7\left(\mathrm{C}-3^{\prime \prime}\right), 28.9\left(\mathrm{C}-4^{\prime \prime}\right)^{* *}, 31.6\left(\mathrm{C}-5^{\prime \prime}\right)^{\psi}, 22.5\left(\mathrm{C}-6^{\prime \prime}\right)^{\psi \psi}, 14.0\left(\mathrm{C}-7^{\prime \prime}\right)^{\dagger}\left({ }^{*}, * *\right.$, ч ${ }^{\Psi},{ }^{\dagger}$ values may be interchanged); HREIMS $(\mathrm{m} / \mathrm{z}): 422.2581\left[\mathrm{C}_{26} \mathrm{H}_{34} \mathrm{~N}_{2} \mathrm{O}_{3}\right.$ calcd. 422.2569]; EIMS $m / z$ (\%): $422\left[\mathrm{M}^{+}\right]$(100), 404 (25), 365 (57), 352 (24), 337 (94), 309 (2), 295 (25), 282 (27), 267 (37), $251(24), 237$ (28).

10,12-Dicapryloyl-11-hydroxy-3-methyl- $\beta$-carboline (30). $27.1 \mathrm{mg}$; mp: 339-340 ${ }^{\circ} \mathrm{C}$; IR $\left(\mathrm{CHCl}_{3}\right) v_{\max }$ $\mathrm{cm}^{-1}$ : $3381.0(\mathrm{OH}$ and indole $\mathrm{N}-\mathrm{H}), 2925.8,2858.3(\mathrm{C}-\mathrm{H}), 1643.2$ (ketone $\left.\mathrm{C}=\mathrm{O}\right), 1579.6,1496.8$, 1377.2 (aromatic $\mathrm{C}=\mathrm{C}), 1202.8(\mathrm{C}-\mathrm{O})$; $\mathrm{UV}(\mathrm{MeOH}) \lambda_{\max } \mathrm{nm}: 365.9,283.8,231.6 ;{ }^{1} \mathrm{H}-\mathrm{NMR}\left(\mathrm{CDCl}_{3}\right)$ : $\delta 8.41(1 \mathrm{H}, \mathrm{d}, J=5.4 \mathrm{~Hz}, \mathrm{H}-5), 7.82(1 \mathrm{H}, \mathrm{d}, J=5.4 \mathrm{~Hz}, \mathrm{H}-6), 8.71(1 \mathrm{H}, \mathrm{s}, \mathrm{H}-9), 2.91$ (3H, s, H-14), $3.15\left(2 \mathrm{H}, \mathrm{t}, J=7.3 \mathrm{~Hz}, \mathrm{H}-2^{\prime}\right), 1.80\left(2 \mathrm{H}\right.$, quintet, $\left.J=7.3 \mathrm{~Hz}, \mathrm{H}-3^{\prime}\right), 1.43\left(2 \mathrm{H}, \mathrm{m}, \mathrm{H}-4^{\prime}\right), 1.30$ (12H, m, H-5', H-6', H-7', H-5', H-6"' and H-7'), 0.88 (3H, t, $\left.J=6.8 \mathrm{~Hz}, \mathrm{H}-8^{\prime}\right), 3.25$ (2H, t, $\left.J=7.3 \mathrm{~Hz}, \mathrm{H}-2^{\prime \prime}\right)$, $1.76\left(2 \mathrm{H}\right.$, quintet, $\left.J=7.3 \mathrm{~Hz}, \mathrm{H}-3^{\prime \prime}\right), 1.41\left(2 \mathrm{H}, \mathrm{m}, \mathrm{H}-4^{\prime \prime}\right), 0.87\left(3 \mathrm{H}, \mathrm{t}, J=6.8 \mathrm{~Hz}, \mathrm{H}-8^{\prime \prime}\right), 14.75(1 \mathrm{H}, \mathrm{s}$, $\mathrm{OH}), 11.36\left(1 \mathrm{H}\right.$, br.s, indole N-H); ${ }^{13} \mathrm{C}-\mathrm{NMR}\left(\mathrm{CDCl}_{3}\right): \delta 135.0(\mathrm{C}-2), 141.0(\mathrm{C}-3), 137.8(\mathrm{C}-5), 112.9$ (C-6), 114.2 (C-7), 131.0 (C-8), 131.1 (C-9), 115.0 (C-10), 167.3 (C-11), 108.9 (C-12), 146.5 (C-13), $18.3(\mathrm{C}-14), 206.9\left(\mathrm{C}-1^{\prime}\right), 38.5\left(\mathrm{C}-2^{\prime}\right), 24.8\left(\mathrm{C}-3^{\prime}\right), 29.3\left(\mathrm{C}-4^{\prime}\right)^{*}, 29.2\left(\mathrm{C}-5^{\prime}\right)^{*}, 31.8\left(\mathrm{C}-6^{\prime}\right)^{* *}, 22.6\left(\mathrm{C}-7^{\prime}\right.$ and C-7"), $14.1\left(\mathrm{C}-8^{\prime}\right), 203.2\left(\mathrm{C}-1^{\prime \prime}\right), 45.3\left(\mathrm{C}-2^{\prime \prime}\right), 24.1\left(\mathrm{C}-3^{\prime \prime}\right), 29.2\left(\mathrm{C}-4^{\prime \prime}\right)^{*}, 29.1\left(\mathrm{C}-5^{\prime \prime}\right)^{*}, 31.7(\mathrm{C}-$ $\left.6^{\prime \prime}\right)^{* *}, 14.1\left(\mathrm{C}-8^{\prime \prime}\right)\left(*, * *\right.$ values may be interchanged); HREIMS $(\mathrm{m} / \mathrm{z}): 450.2898\left[\mathrm{C}_{28} \mathrm{H}_{38} \mathrm{~N}_{2} \mathrm{O}_{3}\right.$ calcd. 450.2882]; EIMS m/z (\%): $450\left[\mathrm{M}^{+}\right]$(98), 432 (43), 403 (6), 379 (83), 366 (51), 351 (100), 323 (2), 295 (32), 282 (26), 267 (32), 251 (14), 237 (21).

Table 2. ${ }^{1} \mathrm{H}-\mathrm{NMR}$ data of 10-propionyl (3), 12-propionyl (11), 10-propionyl-11-hydroxy (18) and 10,12-dipropionyl-11-hydroxy (24) analogues of harmine (1).

\begin{tabular}{ccccc}
\hline $\mathbf{H}$ & $\mathbf{3}$ & $\mathbf{1 1}$ & $\mathbf{1 8}$ & $\mathbf{2 4}$ \\
\hline 5 & $8.21(\mathrm{~d}, 5.7)$ & $8.35(\mathrm{~d}, 5.5)$ & $8.15(\mathrm{~d}, 5.3)$ & $8.42(\mathrm{~d}, 5.3)$ \\
6 & $8.06(\mathrm{~d}, 5.7)$ & $7.82(\mathrm{~d}, 5.5)$ & $7.94(\mathrm{~d}, 5.3)$ & $7.87(\mathrm{~d}, 5.3)$ \\
9 & $8.50(\mathrm{~s})$ & $8.23(\mathrm{~d}, 8.7)$ & $8.81(\mathrm{~s})$ & $8.72(\mathrm{~s})$ \\
10 & - & $6.99(\mathrm{~d}, 8.7)$ & - & - \\
12 & $7.16(\mathrm{~s})$ & - & $6.91(\mathrm{~s})$ & - \\
14 & $2.87(\mathrm{~s})$ & $2.97(\mathrm{~s})$ & $2.77(\mathrm{~s})$ & $2.94(\mathrm{~s})$ \\
$2^{\prime}$ & $3.06(\mathrm{q}, 7.3)$ & $3.19(\mathrm{q}, 7.1)$ & $3.20(\mathrm{q}, 7.2)$ & $3.23(\mathrm{q}, 7.3)$ \\
$3^{\prime}$ & $1.18(\mathrm{t}, 7.3)$ & $1.24(\mathrm{t}, 7.1)$ & $1.25(\mathrm{t}, 7.2)$ & $1.34(\mathrm{t}, 7.3)$ \\
$\mathrm{OCH}_{3}$ & $4.04(\mathrm{~s})$ & $4.08(\mathrm{~s})$ & - & - \\
$2^{\prime \prime}$ & - & - & - & $3.31(\mathrm{q}, 7.3)$ \\
$3^{\prime \prime}$ & - & - & - & $1.25(\mathrm{t}, 7.3)$ \\
OH & - & - & Not observed & $14.69(\mathrm{~s})$ \\
Indole N-H & Not observed & $11.20(\mathrm{br} . \mathrm{s})$ & Not observed & $11.40(\mathrm{br} . \mathrm{s})$ \\
\hline
\end{tabular}

Note: NMRs of $\mathbf{3}$ and $\mathbf{1 8}$ were recorded in $\mathrm{CD}_{3} \mathrm{OD}$, whereas those of $\mathbf{1 1}$ and $\mathbf{2 4}$ were recorded in $\mathrm{CDCl}_{3}$. Assignments were established by interpretation of the ${ }^{1} \mathrm{H}-\mathrm{NMR}, \mathrm{HMQC}, \mathrm{HMBC},{ }^{1} \mathrm{H},{ }^{1} \mathrm{H}$ COSY, ${ }^{1} \mathrm{H},{ }^{1} \mathrm{H}$ TOCSY, and $J$-resolved spectra. Values are in $\delta(\mathrm{ppm})$. Multiplicities and $J$ values (in $\mathrm{Hz}$ ) are in parentheses. 
Table 3. ${ }^{13} \mathrm{C}$-NMR data of 10-propionyl (3), 12-propionyl (11), 10-propionyl-11-hydroxy (18) and 10,12-dipropionyl-11-hydroxy (24) analogues of harmine (1).

\begin{tabular}{ccccc}
\hline $\mathbf{C}$ & $\mathbf{3}$ & $\mathbf{1 1}$ & $\mathbf{1 8}$ & $\mathbf{2 4}$ \\
\hline 2 & 137.0 & 134.3 & 137.4 & 135.0 \\
3 & 142.5 & 138.0 & 142.4 & 141.0 \\
5 & 139.5 & 131.5 & 139.3 & 137.7 \\
6 & 113.6 & 113.7 & 113.8 & 112.8 \\
7 & 116.2 & 116.1 & 115.6 & 114.6 \\
8 & 130.6 & 131.9 & 131.2 & 129.9 \\
9 & 126.0 & 129.4 & 127.1 & 130.6 \\
10 & 124.1 & 107.6 & 116.3 & 114.5 \\
11 & 161.9 & 163.5 & 164.4 & 167.0 \\
12 & 94.6 & 109.9 & 98.5 & 108.9 \\
13 & 146.8 & 144.9 & 147.8 & 146.7 \\
14 & 19.3 & 15.8 & 19.4 & 18.3 \\
$1^{\prime}$ & 204.2 & 203.4 & 208.2 & 206.7 \\
$2^{\prime}$ & 37.5 & 37.0 & 32.2 & 32.4 \\
$3^{\prime}$ & 9.4 & 9.2 & 8.9 & 9.2 \\
OCH $_{3}$ & 56.4 & 56.7 & - & - \\
$1^{\prime \prime}$ & - & - & - & 44.9 \\
$2^{\prime \prime}$ & - & - & - & 9.5 \\
$3^{\prime \prime}$ & - & - & - & 203.5 \\
\hline
\end{tabular}

Note: NMRs of $\mathbf{3}$ and $\mathbf{1 8}$ were recorded in $\mathrm{CD}_{3} \mathrm{OD}$, whereas those of $\mathbf{1 1}$ and $\mathbf{2 4}$ were recorded in $\mathrm{CDCl}_{3}$. Assignments were established by interpretation of the ${ }^{13} \mathrm{C}-\mathrm{NMR}$ (broad band decoupled and DEPT), HMQC, and HMBC spectra.

\section{Acknowledgments}

We would like to thank the Higher Education Commission (HEC), Pakistan for the award of a prestigious Split-Ph.D scholarship to Mr. Syed Nawazish Ali.

\section{References and Notes}

1. Nagendrappa, G. Organic synthesis under solvent-free conditions: An environmentally benign procedure-1. Resonance 2002, 59-68.

2. Brown, A. R.; Hermkens, P. H. H.; Ottenheihm, H. C. J.; Rees, D. C. Solid phase synthesis. Synlett 1998, 817-827.

3. Kaupp, G.; Schmeyers, J.; Boy, J. Waste-free solid-state synthesis with quantitative yield. Chemosphere 2001, 43, 55-61.

4. Tanaka, K.; Toda, F. Solvent-free organic synthesis. Chem. Rev. 2000, 100, 1025-1074. 
5. Olah, G. A. Friedel-Crafts and Related Reactions; Interscience Publishers: New York, 1964; Vol. III.

6. Vogel's Textbook of Practical Organic Chemistry, 4th Ed.; ELBS/Longman: Essex, U.K., 1986; pp. 770-774.

7. Salehi, P.; Khodaei, M. M.; Zolfigol, M. A.; Sirouszadeh, S. Catalytic Friedel-Craft acylation of alkoxybenzenes mediated by aluminum hydrogensulfate in solution and solvent-free conditions. Bull. Chem. Soc. Jpn. 2003, 76, 1863-1864.

8. Smith, K.; El-Hiti, G. A.; Jayne, A. J.; Butters, M. Acetylation of aromatic ethers using acetic anhydride over solid acid catalysts in a solvent free system: Scope of the reaction for substituted ethers. Org. Biomol. Chem. 2003, 1, 1560-1564.

9. Ross, J.; Xiao, J. Friedel-Carafts acylation reactions using metal triflates in ionic liquid. Green Chem. 2002, 4, 129-133.

10. Glasby, J. S. Encyclopedia of the alkaloids; Plenum Press: New York, 1975; Vol. 1, p. 661.

11. Begum, S.; Hassan, S. I.; Siddiqui, B. S.; Ifzal, R.; Perwaiz, S.; Kiran, T.; Shaheen, F.; Ghayur, M. N.; Gilani, A. H. Preparation, structure and spasmolytic activities of some derivatives of harmine series of alkaloids. Nat. Prod. Res. 2006, 20, 213-227.

12. Kusurkar, R. S.; Goswami, S. K. Efficient one-pot synthesis of anti-HIV and anti-tumour betacarbolines. Tetrahedron 2004, 60, 5315-5318.

13. Begum, S.; Hassan, S. I.; Siddiqui, B. S. A rapid, mild, efficient and solvent-free Friedel-Crafts acylation of N-acetyltetrahydroharmine. Synth. Commun. 2005, 35, 23-29.

14. Siddiqui, S. A reinvestigation of the alkaloidal constituents of Peganum harmala. Pak. J. Sci. Ind. Res. 1962, 5, 207-211.

15. Li, J. J. A Collection of Detailed Reaction Mechanisms, 2nd Ed.; Springer-Verlag: BerlinHeidelberg - New York, 2003; p. 149.

16. Norman, R.; Coxon, J. M. Principles of organic synthesis, 3rd Ed.; Chapman \& Hall: London, U.K., 1993; pp. 452-453.

17. Ghiaci, M.; Asghari, J. Friedel-Crafts alkylation and acylation in the absence of solvent. Synth. Commun. 1998, 28, 2213-2220.

18. Coune, C. A.; Denoel, L. J. G. A. J. ${ }^{13}$ C NMR Des Alcaloides Des Strychnos: Les Derives De L'harmane Et De L'usambarensine. Phytochemistry 1980, 19, 2009-2011.

19. Compounds no. 679, 1327, 1335, 1336, 1341 and 1920 in Breitmaier, E.; Haas, G.; Voclter, W. Atlas of Carbon-13 NMR Data; Heyden \& Sons Ltd.: London, 1979.

Sample Availability: Contact the authors.

(C) 2008 by the authors; licensee Molecular Diversity Preservation International, Basel, Switzerland. This article is an open-access article distributed under the terms and conditions of the Creative Commons Attribution license (http://creativecommons.org/licenses/by/3.0/). 